Exotoxins

Bacterial secreted proteins

that damage host cells.

Pelvic inflammatory disease A clinical syndrome where infected fallopian tube tissues are damaged by the host inflammatory response to bacteria

Ectopic pregnancy

A sequela of pelvic inflammatory disease that occurs when a fertilized egg implants anywhere other than the uterine lining, such as in the fallopian tube, which risks organ damage and blood loss.

Department of MicrobiologyImmunology, Northwestern University Feinberg School of Medicine, Chicago, Illinois 60611, USA.

Correspondence to H.S.S h-Seifert@northwestern.edu

doi:10.1038/nrmicro.2017.169 Published online 12 Feb 2018

\title{
Neisseria gonorrhoeae host adaptation and pathogenesis
}

\section{Sarah Jane Quillin and H Steven Seifert}

Abstract | The host-adapted human pathogen Neisseria gonorrhoeae is the causative agent of gonorrhoea. Consistent with its proposed evolution from an ancestral commensal bacterium, $N$. gonorrhoeae has retained features that are common in commensals, but it has also developed unique features that are crucial to its pathogenesis. The continued worldwide incidence of gonorrhoeal infection, coupled with the rising resistance to antimicrobials and the difficulties in controlling the disease in developing countries, highlights the need to better understand the molecular basis of $\mathrm{N}$. gonorrhoeae infection. This knowledge will facilitate disease prevention, surveillance and control, improve diagnostics and may help to facilitate the development of effective vaccines or new therapeutics. In this Review, we discuss sex-related symptomatic gonorrhoeal disease and provide an overview of the bacterial factors that are important for the different stages of pathogenesis, including transmission, colonization and immune evasion, and we discuss the problem of antibiotic resistance.

Neisseria gonorrhoeae (also known as the gonococcus) is the aetiological agent of gonorrhoea, a sexually transmitted infection (STI) that remains a major global public health concern. WHO surveillance of clinical strains of N. gonorrhoeae has identified strains that are resistant to most available antibiotics, highlighting the imminent possibility of widespread untreatable gonorrhoea (BOX 1). Treatment recommendations by the WHO are aimed at both elimination of the organism and prevention of further spread of antimicrobialresistant gonorrhoea ${ }^{1}$. With a worldwide incidence of over 78 million cases each year, uncontrolled transmission and limited treatment options in both low-income countries and poorer communities in developed countries, untreatable gonorrhoea will result in increases in the incidence of and complications from infection ${ }^{1-4}$.

$N$. gonorrhoeae mainly colonizes the genital mucosa but it can also colonize the ocular, nasopharyngeal and anal mucosa ${ }^{5-7}$. Pathology largely results from damage that is caused by the activation of innate immune responses at the sites of colonization, as N. gonorrhoeae does not express potent exotoxins. Complications from untreated, ascending, genital-tract infections in women can include pelvic inflammatory disease, infertility and ectopic pregnancy ${ }^{8}$. Maternal transmission to children during birth can also lead to neonatal blindness9. Untreated N. gonorrhoeae infection can also lead to disseminated gonococcal infection, potentially giving rise to infectious arthritis and endocarditis ${ }^{10}$.
$N$. gonorrhoeae belongs to the genus Neisseria, of which N. gonorrhoeae and Neisseria meningitidis (also known as the meningococcus) are the two pathogenic species, with the latter being a leading cause of bacterial meningitis ${ }^{11}$. In addition, at least eight non-pathogenic commensal Neisseria spp. make up a substantial proportion of the human nasal and oropharyngeal flora ${ }^{12}$. Other Neisseria spp. are able to colonize a range of non-human mammalian and non-mammalian hosts, such as non-human primates, dogs, cats, herbivorous mammals, dolphins, birds and insects ${ }^{13}$. Phylogenetic analyses show that $N$. gonorrhoeae and $N$. meningitidis evolved from a common ancestor but now represent separate lineages that normally occupy distinct niches: the genital mucosa and nasopharyngeal mucosa, respectively ${ }^{14-17}$. Although N. meningitidis can withstand dehydration, survive outside the human host for periods of time and spread via respiratory droplet transmission, $N$. gonorrhoeae is unviable if dehydrated or exposed to non-physiological temperatures. The events that led to the evolution of two separate organisms that are highly similar in their core genome and physiology and yet cause markedly distinct diseases in different locations of the human body are not yet understood. As both the commensal and pathogenic Neisseria spp. occupy the same niches, it is often difficult to differentiate colonization factors from the virulence factors that are necessary to elicit host damage.

As N. gonorrhoeae colonizes genital, rectal and oral mucosa, it expresses a repertoire of factors that enable 


\section{Box 1 | Diagnosis, incidence and epidemiology of Neisseria gonorrhoeae}

Historically, gonorrhoea was diagnosed by a Gram stain of the purulent exudate from a patient showing Gram-negative diplococci among polymorphonuclear leukocytes (neutrophils). This method of diagnosis is still used in the developing world and in remote clinics, but in modern facilities, diagnosis is made using a variety of nucleic-acid-based assays (for example, nucleic acid amplification tests that identify Neisseria gonorrhoeae-specific nucleic acid signatures or, less commonly, antibody-based assays) ${ }^{29}$. Often, diagnosis is confirmed by culture, which requires growing isolates from clinical exudates on N. gonorrhoeae growth medium and examining growth and bacterial morphology. Primary clinical specimens are isolated on non-selective chocolate agar as well as on selective agar containing antimicrobial compounds (such as vancomycin, colistin, trimethoprim lactate, nystatin, and anisomycin or amphotericin B) that stop the growth of other bacteria and fungi ${ }^{151}$. Recently, whole-genome sequencing has been employed to study N. gonorrhoeae epidemiology and the spread of resistance, and it has been discovered that genital Neisseria meningitidis infections are often misdiagnosed as N. gonorrhoeae infections when nucleic-acid-based assays are used ${ }^{152,153}$.

In the developing world, where gonorrhoea is most prevalent, limited resources for public health surveillance, limited self-reporting of sexually transmitted infections (STIs) and barriers to accessing complete medical records can prevent accurate assessment of the burden of gonorrhoea in the population. Nonetheless, in 2012, the WHO reported 78 million cases of gonorrhoea occurring worldwide in people ages $15-49$, which roughly corresponds to a prevalence of $0.6 \%$ among men and $0.8 \%$ among women. As the number of asymptomatic infections and the number of people who do not seek treatment are unknown, it is likely that the actual number of infections is much higher. The highest prevalence for disease is estimated to occur within the western Pacific and African regions. The WHO has developed guidelines for effective diagnosis, treatment and dissemination of information regarding diagnosis and treatment, with the goal of reaching target populations including adults, adolescents aged 10-19, people living with HIV, sex workers, men who have sex with men, and transgender people. Untreated individuals with gonorrhoea are at risk of infertility, pelvic inflammatory disease and, rarely, disseminated gonococcal infection as well as being at risk of transmitting the disease. Gonorrhoea is considered a non-ulcerative STI, like chlamydia and trichomoniasis, and along with other non-ulcerative STIs, people with gonorrhoea have a higher risk of transmitting HIV to their partners owing to increased genital shedding of the virus in people who are co-infected with HIV ${ }^{154,155}$.

replication and survival in these environmental niches and factors that modulate and evade the host immune system. Understanding the mechanisms through which $N$. gonorrhoeae interacts with and evades the host immune system is necessary to facilitate better infection prevention, diagnostic development, surveillance and the development of vaccines and new treatments. In this Review, we discuss the prevalence of asymptomatic infection in both sexes, the main stages of gonococcal pathogenesis, from transmission, colonization, adaptation to environmental conditions and immune evasion, and the rise in antimicrobial resistance. Although $N$. gonorrhoeae and N. meningitidis share many genetic and physiological features, this Review will focus on the host adaptation and pathogenesis of $N$. gonorrhoeae.

\section{Symptomatic and asymptomatic infections}

Differences in the developmental and embryological origins of cells lining the urogenital tracts of men and women have endowed these microenvironments with different surface molecules that act as receptors and co-receptors for N. gonorrhoeae and lead to differences in the mechanisms by which $N$. gonorrhoeae survives in the male and female urogenital niches ${ }^{18}$. In addition, the prevailing dogma in the field is that female genital infections are mostly asymptomatic and male genital infections are mostly symptomatic ${ }^{18-20}$. However, there are many studies showing that asymptomatic infections are common in both sexes ${ }^{21-25}$. The long-held and highly repeated supposition that infections in women are mostly asymptomatic and those in men are symptomatic is mainly based on the fact that overt symptoms (that is, immune cell influx and inflammation) in men are easier to diagnose owing to a purulent exudate from the penis and resultant painful urination. Clinical manifestations in women are more likely to go unnoticed, as inflammation does not occur in the same niche as urination and thus is less likely to be painful. Moreover, symptoms of gonorrhoeal infection in women are more likely to be non-specific, as the vaginal discharge that is caused by neutrophil influx may be mistaken for bacterial vaginosis, yeast infection, hormonal variation in vaginal secretions or normal variability in secretions ${ }^{26}$. Data on the antibody, cytokine and chemokine composition and general magnitude of the inflammatory response in women are sparse and inconclusive. One clinical study of responses to N. gonorrhoeae in individuals and one study of the response of immortalized vaginal and cervical epithelial cells in culture have resulted in different views. Analyses of the cervical mucus of infected and uninfected women found a lack of strong immunoglobulin A1 (IgA1) induction, a slight reduction of IgG levels and an absence of pro-inflammatory interleukin-1 (IL-1), IL-6 and IL-8 cytokines in infected individuals compared with uninfected individuals ${ }^{27}$, suggesting that there was no fulminant inflammatory response to infection. By contrast, analyses of immortalized vaginal and cervical epithelial cells infected with N. gonorrhoeae in vitro showed increased levels of IL-1, IL-6 and IL-8, suggesting that there is an inflammatory cytokine response during infection ${ }^{28}$. Owing to the widespread prevalence of asymptomatic infections in men and women, it is also plausible that a detectable antibody or cytokine response in genital secretions may not result in detectable physiological symptoms. We suggest that the idea that infections in women are normally asymptomatic reflects differences between the anatomy of the urogenital tracts of men and women. To fully understand the epidemiology of gonorrhoea, surveillance and diagnostic tests need to be improved for both sexes ${ }^{25}$ to enable faster responses to gonorrhoea and less expensive treatments (BOX 1).

\section{Transmission}

Transmission is often the most understudied stage of infections, and this is also true for N. gonorrhoeae infections. A successful pathogen must be able to be efficiently transmitted to new hosts, and, as an obligate human colonizer, $N$. gonorrhoeae cannot survive outside the host. Transmission between hosts relies on sexual networks to spread the pathogen from the core, high-risk population in which the majority of infections occur to the fringe, medium-risk group that transmits $N$. gonorrhoeae back to the core group and to the members' partners. High-risk populations include individuals with multiple sexual partners who have unprotected $\operatorname{sex}^{29}$. Individuals are also often unaware that they are 


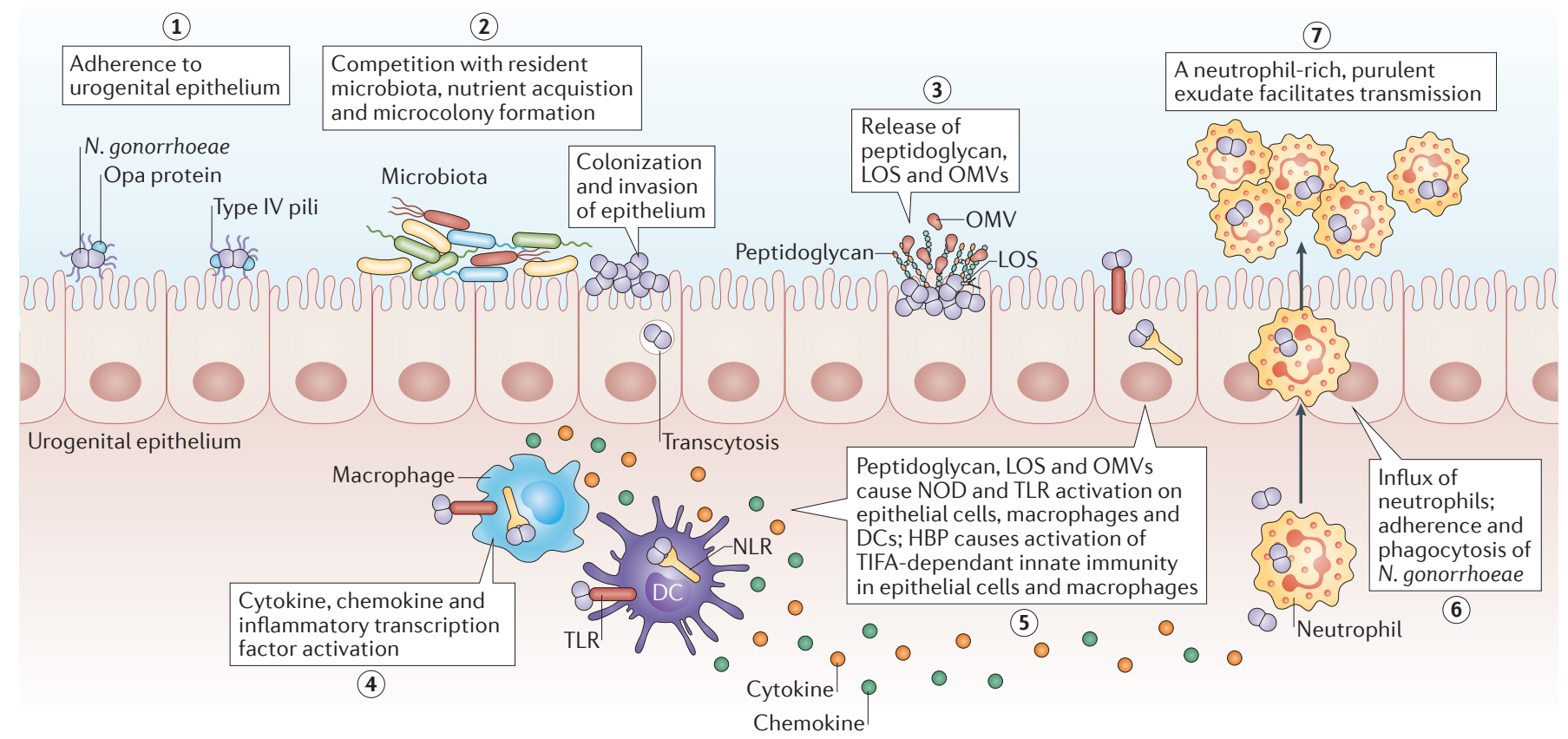

Figure 1 | Overview of Neisseria gonorrhoeae infection. During initial infection, Neisseria gonorrhoeae adheres to host epithelial cells through type IV pili (step 1), which retract and enable epithelial interactions with other prominent surface structures ${ }^{160,161}$. After initial adherence, N. gonorrhoeae replicates and forms microcolonies (step 2), and possibly biofilms ${ }^{34,49}$, and likely competes with the resident microbiota. When colonizing the epithelium, N. gonorrhoeae is capable of invasion and transcytosis. During these initial stages of infection, N. gonorrhoeae releases fragments of peptidoglycan, lipooligosaccharide (LOS) and outer membrane vesicles (OMVs) ${ }^{111,162,163}$ (step 3) that activate Toll-like receptor (TLR) and nucleotide-binding oligomerization domain-containing protein (NOD) signalling in epithelial cells, macrophages and dendritic cells (DCs) ${ }^{111,164,165}$. NOD and TLR signalling from these cells leads to activation of inflammatory transcription factors and the release of cytokines and chemokines (step 4). N. gonorrhoeae also releases heptose-1,7-bisphosphate (HBP), which activates TRAF-interacting protein with FHA domain-containing protein A (TIFA) immunity ${ }^{115}$ (step 5). The release of pro-inflammatory cytokines and chemokines by these innate immune signalling pathways creates cytokine and chemokine gradients that recruit large numbers of polymorphonuclear leukocytes, or neutrophils, to the site of infection (step 6), where they interact with and phagocytose N. gonorrhoeae. The influx of neutrophils makes up a purulent exudate that then facilitates transmission (step 7). NLR, NOD-like receptor; Opa, opacity.

Microcolonies

Collections of bacterial cells that exist as discrete formations.

\section{Antigenic variation}

A reversible process by which a microorganism provides many different versions of a gene product at a frequency higher than the normal mutation rate.

Phase variation

A stochastic form of genetic change that varies gene

expression on/off or up/down. part of a larger sexual network. N. gonorrhoeae attaches to sperm ${ }^{30,31}$ and is easily transmitted from men to their partners through ejaculates, as they contain a high number of bacteria ${ }^{32}$. However, how the efficiency of transmission from women to their partners is maintained is less apparent. The surface of $N$. gonorrhoeae must be free of sialic acid to successfully bind and enter urethral epithelial cells of men, and so it is thought that bacterial sialidases, which are secreted by the cervicovaginal microbiota of women, must first desialylate N. gonorrhoeae lipooligosaccharide (LOS) to enable efficient transmission from women to men $^{33}$.

\section{Establishment of infection}

Adherence, colonization and invasion. Following transmission, $N$. gonorrhoeae establishes contact with the mucosal epithelium to replicate and ultimately be transmitted to new hosts. N. gonorrhoeae is primarily a mucosal colonizer, attaching to various epithelial surfaces. The primary event establishing infection and the first step in pathogenesis (FIGS 1,2) is the bacterial adherence to the epithelium of the mucosa, which is mediated through distinct bacterial surface structures that include type IV pili, opacity (Opa) proteins, LOS and the major outer membrane protein porin (also known as PorB). During initial infection, following initial host cell interaction, $N$. gonorrhoeae attachment and subsequent colonization depends largely on type IV pili forming microcolonies on the epithelial cell surface ${ }^{34}$. Type IV pili are outer membrane structures that are crucial for mediating initial cellular adherence, natural transformation competence, twitching motility and immune evasion through antigenic variation and phase variation ${ }^{35-39}$. Adherence to the epithelial surface and subsequent pilus retraction bring the gonococci close to the cell surface.

Interactions between Opa proteins and carcinoembryonic antigen-related cell adhesion molecule (CEACAM) receptors and other molecules, like heparin sulfate, are important for adherence, and the Opa-CEACAM interaction may be one of the major adherence interactions ${ }^{32,40-42}$. Opa proteins are abundant outer membrane proteins that mediate adherence after initial contact by type IV pili as well as immune evasion by multigene phase variation that results in antigenic variation ${ }^{43-45}$. Type IV pili and Opa proteins are expressed during infection of both women and men $^{40,41,46-48}$ and are considered essential for the colonization of the mucosal epithelium of the genital tract and 


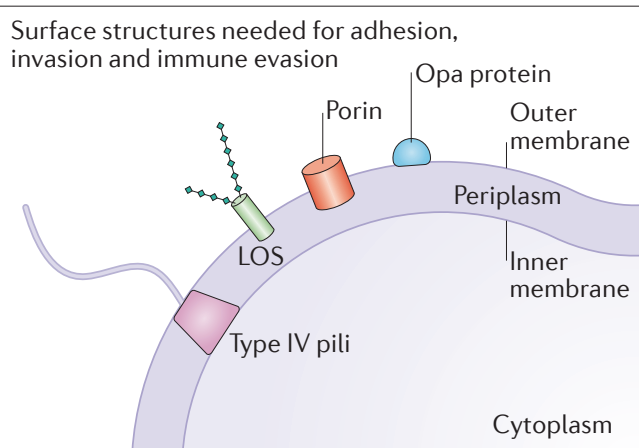

c

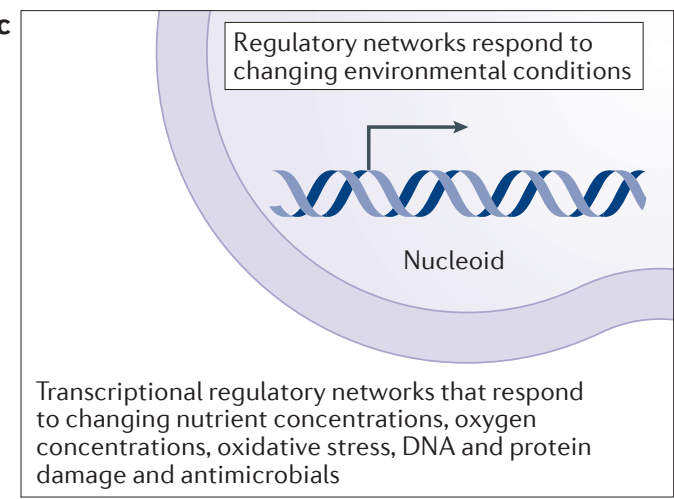

Iron acquisition and efflux of toxic materials

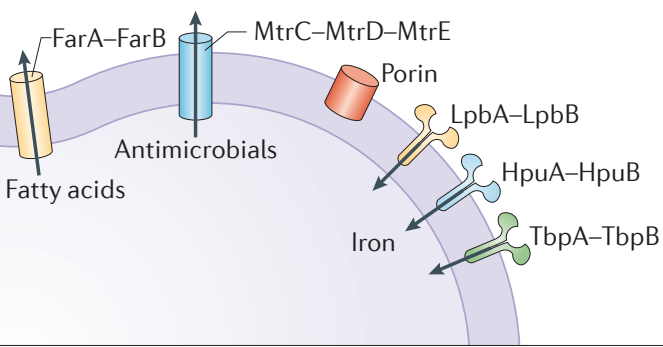

d Endogenous and neutrophil-generated ROS

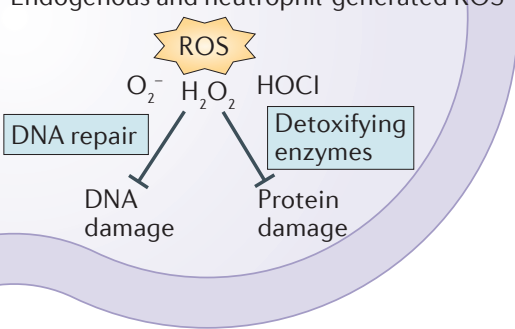

Enzymes quench and detoxify ROS and repair damage to proteins and DNA

\section{Biofilms}

Structured formations of bacterial cells within an extracellular matrix that stick to one another and together on a surface.

\section{C4b-binding protein} (C4BP). A classical complement pathway regulatory protein, akin to factor $\mathrm{H}$ in the alternative pathway, that regulates complement activation on host cells.

Factor $\mathrm{H}$

A control protein of the alternative complement pathway that binds $\mathrm{C} 3 \mathrm{~b}$, displacing activated factor $\mathrm{Bb}$ and enabling cleavage and subsequent inactivation of $\mathrm{C} 3 \mathrm{~b}$ by factor $\mathrm{l}$-induced conversion to iC3b.

Oxidative burst

An antimicrobial response through the release of reactive oxygen species from host cells.

Transcytosis

The transit of the cellular

epithelium by a bacterium

Figure 2 | Overview of Neisseria gonorrhoeae pathogenesis factors. As a host-restricted pathogen, Neisseria gonorrhoeae encodes a relatively small repertoire of pathogenesis and colonization factors compared with other Gram-negative bacteria ${ }^{166}$ a $\mid$ N. gonorrhoeae uses an array of surface structures to adhere to host cells, occasionally invade host cells and evade the immune system ${ }^{18,142,143}$. These surface structures include type IV pili, lipooligosaccharide (LOS), porin, and opacity (Opa) proteins. b | Efflux pumps protect N. gonorrhoeae from antimicrobials and fatty acid stress, and membrane transporters allow N. gonorrhoeae to co-opt nutrients from the surrounding environment ${ }^{66,141,167-169}$. The pump FarA-FarB controls fatty acid transport, while the pump MtrC-MtrD-MtrE controls antimicrobial peptides. The membrane transport complexes LpbA-LpbB, HpuA-HpuB and TbpA-TbpB contribute to iron transport and iron homeostasis. c|A set of transcriptional regulators, discussed in detail in the main text and FIG. 3, induce transcriptional programmes to adapt and respond to changing environmental conditions during infection $82,127,170-173$. The regulons that respond to iron levels, oxidative conditions and oxygen concentration are co-regulated and interconnected. $\mathbf{d}$ | Protective enzymes like catalase and peptide methionine sulfoxide reductase (MsrA-MsrB) detoxify reactive oxygen species (ROS), such as superoxide anion $\left(\mathrm{O}_{2}^{-}\right)$, hydrogen peroxide $\left(\mathrm{H}_{2} \mathrm{O}_{2}\right)$ and hypochlorous acid $(\mathrm{HOCl})$, that are generated endogenously and by neutrophils ${ }^{86}$.

other sites of infection (FIG. 2a). N. gonorrhoeae can form biofilms on abiotic surfaces and epithelial cells in vitro ${ }^{49,50}$; however, the precise role of biofilms during infection remains to be determined ${ }^{50-52}$; it is not known whether stable mucosal colonization during infection is mediated by microcolonies, biofilms or a combination of both.

The prominent surface factors porin and LOS also affect colonization. Porin is a nutrient channel, is one of the most abundant gonococcal outer membrane proteins, binds complement factors C4b-binding protein (C4BP) and factor $\mathrm{H}$ and suppresses the neutrophil oxidative burst and neutrophil apoptosis ${ }^{53}$. LOS is localized to the outer leaflet of the outer membrane and is similar in structure to the ubiquitous bacterial lipopolysaccharides, although LOS lacks the $\mathrm{O}$-antigen polymer ${ }^{54}$. LOS is important for adherence and invasion of host cells; variations in LOS affect immune cell recognition, and sialylation of LOS affects serum resistance through complement evasion and host transmission ${ }^{54-56}$. In addition to colonization of the mucosal epithelium, $N$. gonorrhoeae can invade epithelial cells. Although less is known about mucosal cell invasion than surface colonization, it has been shown that $N$. gonorrhoeae invades non-ciliated cervical epithelial cells and the urethral epithelial cells of men when LOS is desialylated ${ }^{18}$. It is thought that the interaction between LOS and the asialoglycoprotein receptors promotes epithelial invasion in the urethra of men, whereas complement receptor 3 (CR3) serves as the receptor that mediates invasion in the lower cervical genital tract and lutropinchoriogonadotropic hormone receptor serves as the receptor in the endometrium and in fallopian environments. This invasion of the epithelium and the resultant transcytosis of the epithelium could lead to disseminated gonococcal infection, but the relevance of epithelial cell invasion and transcytosis to uncomplicated infections is less clear. The differences in receptors that mediate epithelial invasion highlight the complex, multifaceted nature of tissues lining the genital tract, which is a central part of the difficulty in establishing appropriate animal and tissue culture models to study a host-restricted pathogen (BOX 2). 


\section{Nutritional immunity \\ The ability of the host to sequester important nutrients during infection. \\ Siderophores \\ Low molecular mass \\ iron-binding chemical \\ compounds secreted by \\ bacteria to chelate iron for \\ subsequent uptake into the \\ bacterial cell.}

Microaerophilic

Environments where oxygen concentration is limited but not zero
Growth and metabolism. Once N. gonorrhoeae adheres to the mucosal epithelium, efficient colonization requires extracellular bacterial replication and nutrient acquisition from the surrounding extracellular milieu. It has not been thoroughly determined which microenvironments are encountered during colonization, and thus, the exact nutrient composition of each ecological niche that N. gonorrhoeae may inhabit during urogenital, rectal and oropharyngeal infection is unknown. In laboratory culture, $N$. gonorrhoeae has complex media requirements. Specifically, bacteria cannot grow in culture without a supplemented source of glucose, glutamine, thiamine, phosphate, iron and carbon dioxide $^{57-59}$. In order to meet its nutritional requirements, $N$. gonorrhoeae must interact and possibly compete with resident microbiota for available nutrients ${ }^{60-62}$ (FIG. 1). Indeed, $N$. gonorrhoeae must acquire nutrients like iron, zinc and manganese (FIG. 3) that are limited by the human host as a defence against bacterial pathogens in a process termed nutritional immunity ${ }^{63,64}$. As Neisseria spp. lack siderophores, $N$. gonorrhoeae scavenges iron directly from host-bound complexes, obtaining metals through a series of membrane transport complexes by transporting them into the bacterial cell ${ }^{65-68}$ (FIG. 2). Finally, the influx of neutrophils that occurs during symptomatic colonization (FIG. 1) may promote nutrient acquisition by causing leakage of serum components and tissue damage and by exposing $N$. gonorrhoeae to intracellular nutrient pools following phagocytosis, thus providing nutrients for bacterial growth ${ }^{69-74}$.

Regulatory networks. In order to survive and replicate, $N$. gonorrhoeae must adapt to changing environmental conditions within the host genital tract, rectum and oropharynx (FIG. 3). The limitations of current N. gonorrhoeae experimental models have prevented gains in

\section{Box 2 | Neisseria gonorrhoeae infection models}

As Neisseria gonorrhoeae has evolved to survive and persist only within the human body, there are limitations to understanding the complexities of the environmental challenges that the bacterium encounters during infection in humans. These limitations may include, but are not limited to, the specific types and local concentrations of specific nutrients (for example, iron, manganese, zinc, glucose, lactate and pyruvate) that are present during infection, the oxygen concentration at different sites during colonization, the heterogeneity of cytokine and chemokine responses among individuals and their immune cell profiles, and the composition of local microbiota that is encountered during infection. Currently, the interaction of N. gonorrhoeae with specific types of host cells (epithelial cells, endothelial cells, neutrophils and macrophages) can be studied ex vivo by using immortalized or primary cell lines. However, immortalized cell lines do not always model human tissues, and the heterogeneity of primary human cells can introduce variability into these studies ${ }^{114}$. The most well-developed mouse model is the oestradiol-treated vaginal infection model ${ }^{156}$, but this model is limited to addressing certain questions - specifically, which factors affect short-term colonization or survival of $\mathrm{N}$. gonorrhoeae in the murine vaginal tract - owing to its lack of receptors (complement receptor 3 (CR3), CD46 and carcinoembryonic antigen-related cell adhesion molecules (CEACAMs)) that are required for bacterial adherence to and subsequent colonization of the mouse mucosal epithelium and other differences in host physiology. The ongoing development of transgenic mouse models that express human receptors will enable the study of $N$. gonorrhoeae pathogenesis; however, these models may not be able to replicate all the factors that are required for human infection ${ }^{157-159}$. specific knowledge of the $\mathrm{pH}$, and nutrient and oxygen concentrations in the varying ecological niches of the genital mucosa; however, the range of adaptive mechanisms that the bacterium has acquired and maintained over evolutionary time indicates the main contributing environmental changes that may affect survival during infection. These mechanisms regulate global transcriptional changes through transcriptional regulators and two-component systems (FICS 2c,3), translational regulation through regulatory small RNAs (sRNAs) and clonal variation through phase variation.

For specific environmental variables (for example, metal availability, oxygen concentrations, reactive oxygen species, protein misfolding, membrane stress and the presence of antimicrobial peptides (FIG. 2b-d)), $N$. gonorrhoeae utilizes an array of responsive transcriptional factors to activate and repress small-scale and large-scale adaptive transcriptional programmes (FIG. 3). During infection, $N$. gonorrhoeae encounters antimicrobial peptides. Transcriptional regulators MtrR, MtrA and MpeR contribute to regulation of an antimicrobial efflux pump, MtrC-MtrD-MtrE, which exports antimicrobial peptides $^{75-78}$. RNA polymerase- $\sigma$ factor RpoH maintains protein homeostasis, and the two-component system MisR-MisS responds to membrane stress ${ }^{79}$. Portions of the human genital mucosa present a microaerophilic environment, but different subcellular locations may vary in oxygen concentration. N. gonorrhoeae is capable of either aerobic or anaerobic respiration controlled through a truncated denitrification pathway that is regulated by copper-containing nitrite reductase (AniA) and nitric oxide reductase subunit $\mathrm{B}(\mathrm{NorB})^{80,81}$. The oxygensensing fumarate and nitrate reduction regulator protein (Fnr) is required for activation of this pathway, in addition to the two-component system containing nitrate/nitrite sensor protein NarQ and nitrate/nitrite response regulator $\mathrm{NarP}^{82}$. Intracellular iron homeostasis is maintained via the ferric uptake regulation protein (Fur), and there is substantial overlap between the regulons responsive to iron, anaerobic conditions and oxidation, highlighting the fine-tuned and interconnected nature of adaptive regulatory networks in $N$. gonorrhoeae ${ }^{83-86}$.

$N$. gonorrhoeae encodes a specialized repertoire of 34 putative transcriptional regulators, two-component systems and sRNAs. These systems are relatively small in number compared with the $\sim 200$ transcriptional regulators, two-component systems and sRNAs found in Escherichia coli. The small number of these regulons in N. gonorrhoeae is likely due to the organism's long evolutionary history solely colonizing human hosts, thereby limiting the diversity of environmental conditions it may encounter and regulons it may require to survive. In addition, a large number of N. gonorrhoeae genes are stochastically modulated by phase variation ${ }^{87}$, likely reducing the need for transcriptional regulation. During phase variation, Neisseria spp. modulate protein production. Protein production may be altered through changes in transcription efficiency or changes in translation efficiency. Transcription efficiency is altered by varying the numbers of polynucleotide repeat sequences in genes, thus tuning the levels of gene expression. 


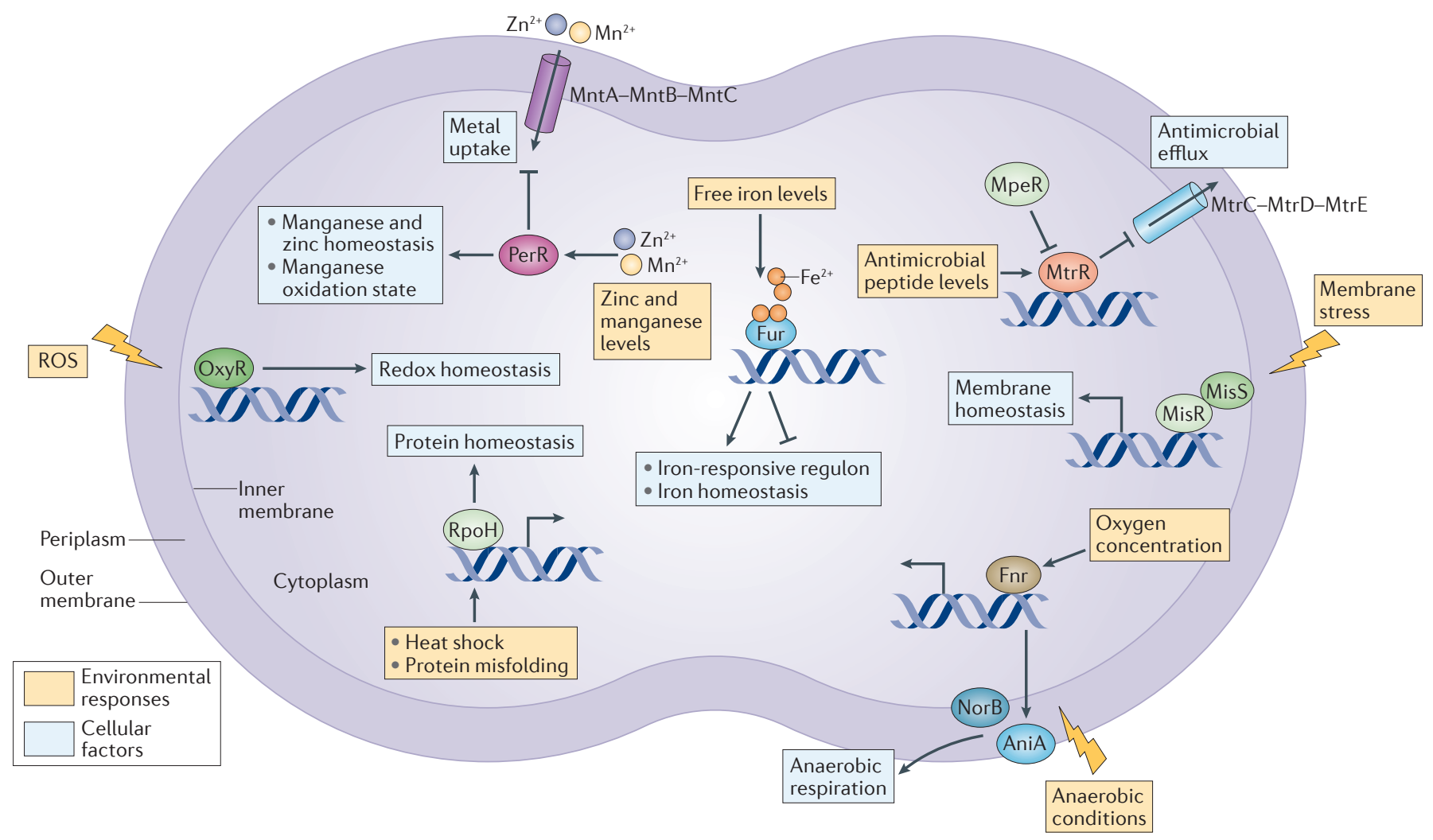

Figure 3 | Overview of transcriptional regulatory factors of Neisseria gonorrhoeae. To adapt to a changing urogenital, rectal and oropharyngeal environment during infection, Neisseria gonorrhoeae has moderately few regulatory networks. N. gonorrhoeae has regulators that specifically respond to metal availability, antimicrobial peptides, oxygen availability, membrane stress and protein misfolding. These systems are often overlapping, particularly at the level of iron and oxygen availability. Transcriptional regulator MpeR is known to repress the antimicrobial efflux pump operon repressor MtrR; both regulators mediate antimicrobial peptide efflux ${ }^{76,78}$. The two-component regulatory system composed of sensor histidine kinase and response regulator (MisR-MisS) responds to membrane perturbations and controls membrane homeostasis ${ }^{174}$. The oxygen-sensor fumarate and nitrate reduction regulator protein (Fnr) responds to oxygen concentrations and contributes to regulation of genes encoding copper-containing nitrite reductase (AniA) and nitric oxide reductase subunit B (NorB), which control a denitrification system required for anaerobic respiration ${ }^{82,171}$. Iron-response master regulator ferric uptake regulation protein (Fur) responds to fluctuating iron levels and controls iron homeostasis under iron-replete and iron-starved conditions ${ }^{175}$. Peroxide-responsive repressor (PerR) responds to fluctuating zinc and manganese levels, controlling metal influx through MntA-MntB-MntC and zinc and manganese homeostasis ${ }^{172}$. RNA polymerase- $\sigma$ factor $\mathrm{RpoH}$ responds to heat shock and protein misfolding and controls a regulon that maintains protein-folding homeostasis ${ }^{173}$. Oxidative stress regulatory protein OxyR responds to the presence of reactive oxygen species (ROS) and maintains redox homeostasis ${ }^{176}$.

Translation efficiency is altered through changes in the gene coding sequence, like the introduction of stop codons or repeat sequences, thus tuning protein production. N. gonorrhoeae is estimated to phase vary over 100 genes that encode a variety of gene products ${ }^{88}$. Phase variation presumably provides different advantages to the numerous subpopulations that result during colonization. The opa gene family is the most wellcharacterized, phase-variable system both mechanistically and functionally ${ }^{89}$. Opa phase variation occurs through changes in the number of CTCTT repeats in the leader peptide sequence of each of $11 \mathrm{opa}$ alleles in the chromosome, resulting in altered expression of these genes and affecting host cell adherence and neutrophil stimulation. The ability of N. gonorrhoeae to generate a repertoire of different phenotypes within a clonal lineage promotes long-term adaptation on the bacterial population level. In addition to phase variation of Opa proteins, $N$. gonorrhoeae also encodes a phase-variable methyltransferase, ModA13, that switches between an on and off state of gene expression, regulating changes in polynucleotide repeats that occur during replication. Differences in the amount of ModA13 activity result in altered methylation patterns of promoters and expression levels of various genes. This phenomenon is called the ModA13 phasevarion and influences virulence factor gene expression and biofilm formation ${ }^{90-94}$. $N$. gonorrhoeae proteins may also be globally regulated by post-translational modifications, and acetylation has been shown to affect numerous pathways, anaerobic growth and the ability to form biofilms ${ }^{95}$. Lastly, it is known that $N$. gonorrhoeae expresses sRNAs. Although many $N$. gonorrhoeae regulatory sRNAs have not been characterized mechanistically, it is known that the sRNA 
$\mathrm{NrrF}$ is transcribed in response to iron availability and controls a small regulon ${ }^{96}$ and that another sRNA, FnrS, controls a regulon of four genes in response to anaerobic conditions ${ }^{84}$.

\section{Interactions with the host immune system}

Interactions with complement. The alternative complement pathway and the classical complement pathway are major arms of the innate immune system that converge at the level of protein complement C3, which can lead to the deposition of opsonin C3b to facilitate bacterial phagocytosis and kill invading pathogens through the formation of membrane attack complexes. The alternative and classical complement pathways are activated by the presence of invading microorganisms; the former is activated by non-specific tissue damage or microorganism binding, and the latter is activated through IgG and IgM antibody deposition that leads to clearance by the immune system. Activation of both pathways converges on cleavage and activation of $\mathrm{C} 3$, which is crucial for maintaining a cascade that results in the assembly of membrane attack complexes (transmembrane pores that form on bacterial surfaces), causing lysis and cell death.

The ability of $N$. gonorrhoeae to evade recognition and attack from the human complement system is a major feature of host adaptation by this species, which is highlighted by the observation that $N$. gonorrhoeae resists the action of the human complement system but is sensitive to animal complement systems ${ }^{97}$. Patients with complement deficiencies have been found to have a higher risk of systemic $N$. gonorrhoeae infection ${ }^{98,99}$. In both the cervical epithelium and human serum, the alternative and classical complement pathways respond to $N$. gonorrhoeae infection by initiating the complement cascade to opsonize invading bacteria ${ }^{18,100-102}$. Studies in vitro have shown that $N$. gonorrhoeae interacts with several complement components ${ }^{103} . N$. gonorrhoeae evades complement-mediated killing through two general mechanisms: by binding to and inactivating complement cascade components and preventing membrane attack complex formation, and by presenting itself as part of the host by expressing molecules found in the host on the bacterial surface and binding to complement regulatory proteins (FIG. 4a).

In the first mechanism, $N$. gonorrhoeae inactivates the complement cascade through factor I. C3b binds to gonococcal LOS through lipid A and is rapidly inactivated by factor I-mediated cleavage to $\mathrm{iC} 3 \mathrm{~b}$, thus inactivating the complement cascade ${ }^{101}$ (FIG. 4a). In addition, in the cervical epithelium, $N$. gonorrhoeae binds to the alternative complement pathway receptor CR3 and the receptor for $\mathrm{iC} 3 \mathrm{~b}$, which is thought to facilitate epithelial cell invasion ${ }^{100,102}$.

In the second mechanism, $N$. gonorrhoeae shields itself from complement recognition, thus subverting complement activation in both the cervical epithelium and human serum. In the cervical epithelium, N. gonorrhoeae binds the alternative complement pathway regulator factor $\mathrm{H}$ through sialylated LOS and porin (FIG. 4a). Normally, factor $\mathrm{H}$ acts as an alternative complement pathway regulatory protein that binds sialylated cell structures to protect cells, as host structures bound to factor $\mathrm{H}$ are considered self and not targeted for opsonization and lysis ${ }^{104,105}$. In the serum, $N$. gonorrhoeae can bind the classical complement pathway regulator C4BP, a molecule that has a similar function to factor $\mathrm{H}$, to the porin ${ }^{106}$. Moreover, N. gonorrhoeae can bind complement regulatory factor CD46 through the pilus, though the role of this interaction in pathogenesis is not fully defined ${ }^{107}$ (FIG. 4a). Without the ability to avoid complement recognition and killing, $N$. gonorrhoeae would not be able to effectively colonize epithelial mucosa and grow, as shown by its sensitivity to killing by animal complement components.

Immune cell detection and signalling. Owing to the lack of surveillance and difficulty in diagnosing asymptomatic gonorrhoea, little is known about how the immune system responds to $N$. gonorrhoeae during asymptomatic infection, though these infections likely represent a high and likely underreported proportion of infections. Symptomatic infection stimulates the release of pro-inflammatory cytokines and chemokines (IL-6, IL-8, IL-1B, IL-17, interferon- $\gamma$ (IFN $\gamma$ ) and the cytokineexpression controlling transcription factor nuclear factor- $\kappa \mathrm{B}(\mathrm{NF}-\kappa \mathrm{B})$ ), causing an influx of neutrophils to the site of infection and potentially causing inflammatory damage within the epithelial mucosa ${ }^{74,108-110}$ (FIG. 1). During colonization, bacterial factors like LOS and peptidoglycan are present and capable of triggering their cognate innate immune sensors Toll-like receptor 2 (TLR2), TLR4, nucleotide-binding oligomerization domain-containing protein 1 (NOD1) and NOD2, all of which induce signalling of the host innate immune system $^{111-113}$ (FIG. 1). In addition, detection by immune sentinel cells like macrophages and dendritic cells $s^{55,114}$ releases a gradient of cytokines and chemokines, including IL-6, IL-8, IL-1B, IL-17 and IFN $\gamma$, thus resulting in an influx of neutrophils. N. gonorrhoeae has also been shown to release heptose-1,7-bisphosphate, a metabolic intermediate that acts as a pathogen-associated molecular pattern to trigger TRAF-interacting protein with FHA domain-containing protein A (TIFA)-dependent innate immunity ${ }^{115}$ (FIG. 1). Moreover, N. gonorrhoeae can survive within macrophages by modulating apoptosis and cytokine production ${ }^{114}$ and may polarize macrophages in a way that suppresses $\mathrm{T}$ cell proliferation ${ }^{116}$ (FIG. 4b).

As discussed earlier in this Review, N. gonorrhoeae colonization may result in either symptomatic or asymptomatic infection. Symptomatic infection occurs when there is sufficient neutrophil influx into the site of infection to produce a purulent exudate. Although it is known that the presence of a purulent exudate is a result of bacterial innate immune stimulation that causes cytokine and chemokine signalling and neutrophil influx, it is not known whether asymptomatic patients also recruit neutrophils to the site of infection. It is possible that $N$. gonorrhoeae recruits neutrophils in numbers that are insufficient in some people to produce observable symptoms, but it is also possible that neutrophils are not recruited to the site of infection during asymptomatic colonization. 


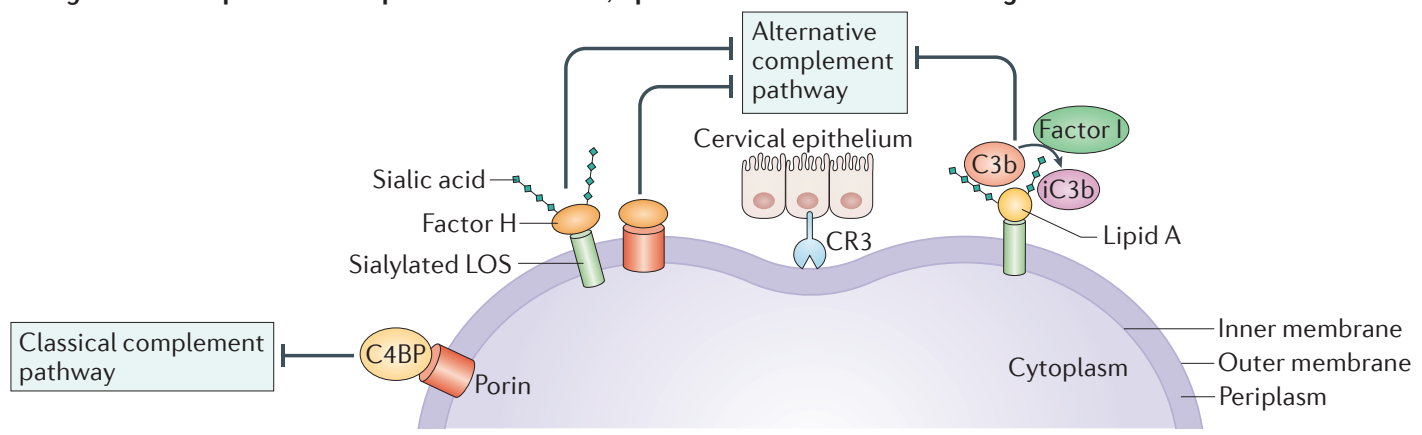

b N. gonorrhoeae modulates the activities of macrophages, DCs and neutrophils

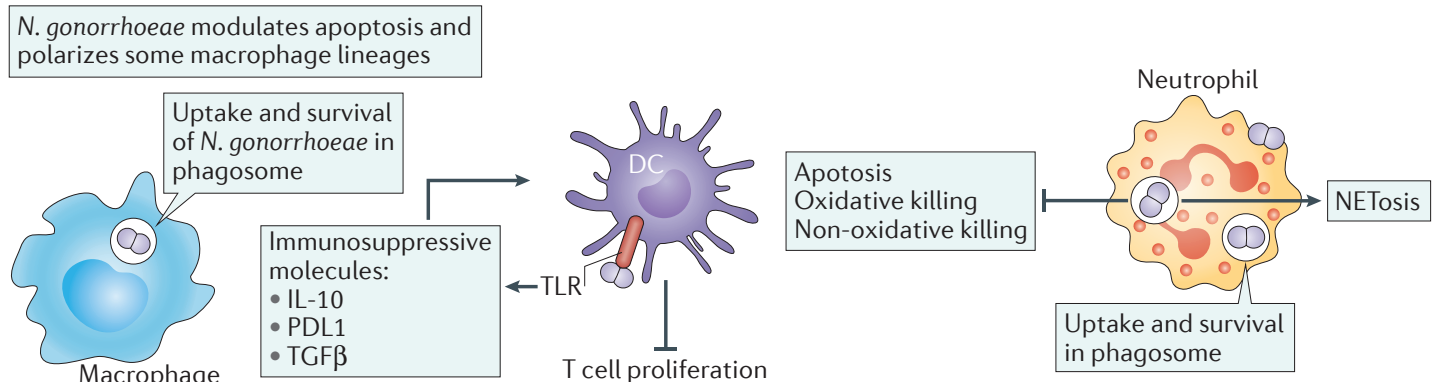

c N. gonorrhoeae modulates T cell function and varies its surface components to avoid the adaptive immune system

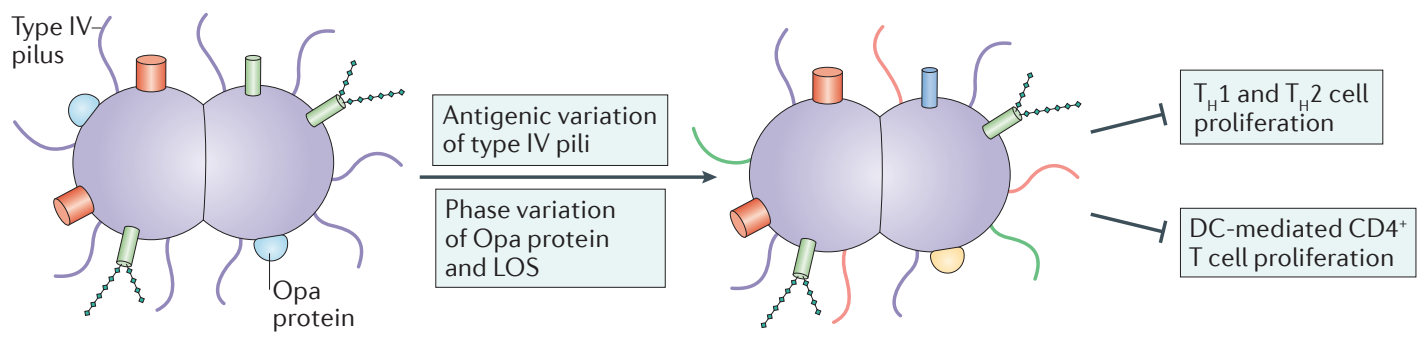

Figure 4 | Neisseria gonorrhoeae evades and modulates the innate and adaptive immune systems. a | During infection, both the alternative and classical complement pathways may be activated by Neisseria gonorrhoeae. N. gonorrhoeae binds complement proteins to prevent opsonization and killing by membrane attack complexes ${ }^{18}$ and sialylates its lipooligosaccharide (LOS) to hide from the complement system ${ }^{177}$. N. gonorrhoeae binds host factor $\mathrm{H}$ and $\mathrm{C} 4 \mathrm{~b}$-binding protein (C4BP), becoming serum resistant by presenting as self and by shielding itself from complement recognition ${ }^{97,178} \mathrm{~N}$. gonorrhoeae also binds to the alternative complement pathway receptor complement receptor 3 (CR3) and the receptor for $\mathrm{iC} 3 \mathrm{~b}$, a process thought to aid in epithelial cell invasion ${ }^{100}$. N. gonorrhoeae binds $\mathrm{C} 3 \mathrm{~b}$ through lipid $\mathrm{A}$ on its $\mathrm{LOS}$, rapidly inactivating $\mathrm{C} 3 \mathrm{~b}$ by factor $\mathrm{l}$-induced conversion to $\mathrm{iC} 3 \mathrm{~b}^{179} . \mathbf{b} \mid \mathrm{N}$. gonorrhoeae is able to survive in and around macrophages and neutrophils during infection and modulate the immune-activating properties of dendritic cells (DCs) ${ }^{114,116,122,124,136,164,180}$. In macrophages, N. gonorrhoeae is able to survive inside the phagosome and modulate apoptosis and production of inflammatory cytokines ${ }^{114}$. The bacterium polarizes macrophages, resulting in macrophages that are less capable of T cell activation ${ }^{116}$, and, similarly, DCs exposed to N. gonorrhoeae are less capable of stimulating T cell proliferation ${ }^{136}$. The interactions of $N$. gonorrhoeae and neutrophils are complex and are discussed in detail in the main text. c $\mid N$. gonorrhoeae infection does not generate immunological memory, owing to the ability of $N$. gonorrhoeae to antigenically and phase vary its surface structures, including type IV pili, opacity (Opa) proteins and LOS. In addition, $N$. gonorrhoeae modulates the adaptive immune response by suppressing Thelper $1\left(T_{H} 1\right)$ and $T_{H} 2$ cell proliferation and subsequent activation by influencing cytokine production ${ }^{134,135}$. IL-10, interleukin-10; NETosis, cell death caused by neutrophil extracellular traps (NETs); PDL1, programmed cell death 1 ligand 1; TGF $\beta$, transforming growth factor- $\beta$; TLR, Toll-like receptor.

Differences in N. gonorrhoeae Opa variants and their propensity for CEACAM binding and immune stimulation may explain the heterogeneity of symptoms in infected individuals. Opa-CAECAM interactions determine neutrophil adhesion, phagocytosis and stimulation of the oxidative burst. The $N$. gonorrhoeae cell surface may lack Opa proteins entirely (Opa-less), express one Opa variant only or express a combination of many opa alleles. During human infection, there is variability of Opa expression in N. gonorrhoeae ranging from multiple Opa-expressing to Opa-less strains, and Opa expression is correlated with the menstrual cycle ${ }^{47}$. 
It is not known whether particular Opa expression patterns predominate during asymptomatic or symptomatic infection. Although CEACAM1, CEACAM3, CEACAM5 and CEACAM6 bind to N. gonorrhoeae Opa variants, only CAECAM1, CEACAM3 and CEACAM5 are expressed on neutrophils ${ }^{117}$. The Opa-CAECAM3 interaction is the only one known to stimulate a bactericidal neutrophil oxidative burst. Although the underlying mechanism for why some infections elicit observable symptoms and some do not is unknown, it has been proposed that a subset of the bacterial population capable of Opa-CEACAM3 binding induces killing by neutrophils of a sufficient number of bacteria to prevent massive neutrophil influx and an observable purulent exudate. There is evidence that neutrophils use CEACAM3 as a decoy receptor, as bacterial contact with the CEACAM family of receptors enables colonization of other cells types but on neutrophils enables capture of Opa-expressing variants for subsequent phagocytosis, neutrophil activation and killing ${ }^{118,119}$.

Neutrophil infiltration to sites of infection. Similarly, although it is known that $N$. gonorrhoeae is able to both evade and modulate host immune responses, there is disagreement as to whether neutrophil recruitment ultimately serves to benefit the host, pathogen or both. One hypothesis is that N. gonorrhoeae aims to remain undetected by the immune system because immune stimulation ultimately benefits the host. This hypothesis is supported by the observation of prevalent asymptomatic infections in humans, the observation that asymptomatic partners are infectious and data showing that bacterial shedding does not correlate with neutrophil infiltration in mice ${ }^{120}$. An alternative hypothesis is that neutrophil inflammation at sites of infection serves primarily to benefit the pathogen, largely through facilitating transmission. In this scenario, the neutrophil exudate carries live, replicating bacteria, analogous to a Trojan horse $\mathrm{f}^{74}$. Data supporting the argument that neutrophil inflammation benefits the pathogen show that, although the presence of N. gonorrhoeae recruits neutrophils to the site of infection, most bacteria (except those expressing Opa variants that engage CEACAM3 on neutrophils) are able to survive and replicate inside and outside of neutrophils ${ }^{86,121-127}$. In this scenario, it is possible that the transfer of infected neutrophils between partners through sexual contact with purulent exudate is a mechanism that the bacterium exploits for efficient transmission from women to their partners ${ }^{74}$. Several components of seminal plasma, like lactoferrin ${ }^{77}$, are chemoattractants for neutrophils ${ }^{128}$. However, testing any transmission hypothesis is difficult because human transmission studies are unethical and there is no animal model that accurately models sexual transmission. The ambiguity of the role of neutrophils in disease pathogenesis stems from the heterogeneous nature of interactions between $N$. gonorrhoeae and neutrophils, a lack of knowledge regarding what causes some infections to be symptomatic whereas others show symptoms, and limitations to experimental models. BOX 2 addresses the usefulness and limitations of current models that are used to study mechanisms of N. gonorrhoeae pathogenesis. As this is a system in equilibrium, it is probable that specific hostpathogen interactions sway the equilibrium in either direction, ultimately determining whether neutrophil influx benefits the host or the pathogen for each individual infection.

Adaptive immunity and issues with vaccine development. It is known that individuals who have been treated for gonorrhoea can be repeatedly infected with no development of immunological memory. An experimental gonococcal infection model study in men showed that initial infection failed to provide protection against repeated infection with the identical strain within 21 days of initial infection ${ }^{129}$. N. gonorrhoeae evades the adaptive arm of the human immune system by several mechanisms. N. gonorrhoeae undergoes antigenic and phase variation of the surface-exposed type IV pili, Opa proteins and LOS to escape immunity ${ }^{45,48,130}$ (FIG. 4C). The carbohydrate structures of LOS also have a role in immune evasion by mimicking host molecules. Many N. gonorrhoeae LOSs show cross reactivity with antibodies that recognize human glycosphingolipid surface antigens, particularly on human erythrocytes, mimicking human surface antigens and contributing to the difficulty of vaccine development ${ }^{131-133}$. In addition, N. gonorrhoeae has been shown to actively suppress the adaptive immune response by modulating IL-10 production from mouse iliac lymph node cells, $\mathrm{CD} 4^{+} \mathrm{T}$ cells and genital tract explants by modulating transforming growth factor- $\beta$ (TGF $\beta$ ) cytokine production in BALB/c mouse vaginal cells and the type 1 regulatory $\mathrm{T}$ cell activity of $\mathrm{CD} 4^{+} \mathrm{T}$ cells, thus preventing $\mathrm{T}$ helper 1 and $\mathrm{T}$ helper 2 cell development ${ }^{134,135}$. Moreover, dendritic cells that have been exposed to $N$. gonorrhoeae are no longer capable of inducing CD4 ${ }^{+} \mathrm{T}$ cell proliferation ${ }^{136}$. Whereas $N$. gonorrhoeae stimulates a large innate immune response from the human host and suppresses the adaptive immune response, these interactions of $N$. gonorrhoeae on both arms of the immune system can extend infections and enable repeated infections in the high-risk group of the population ${ }^{137}$.

Strategies that have searched for protective antigens by comparing infected individuals have been unsuccessful for $N$. gonorrhoeae vaccine development because natural protection is uncommon (if it exists at all). The ability of N. gonorrhoeae to antigenically and phase vary multiple surface proteins has reduced the number of viable vaccine antigen candidates. Vaccine development has also been hampered by the lack of a global systematic vaccine antigen analysis where many antigen candidates are consistently tested in a high-throughput manner as compared with the few that have been tested in disparate experiments. Purified pili and killed whole cells have been tested, but neither has resulted in a viable vaccine ${ }^{138}$. The lack of viable vaccine antigen candidates combined with limitations to the current animal models has also impeded progress. A recently published study from New Zealand, wherein young adults were inoculated 
Outer membrane vesicle A membrane-bound vesicle that is secreted from the bacterial envelope and can contain a variety of cellular material. with a group B outer membrane vesicle meningococcal vaccine, showed reduced rates of gonorrhoea in that population ${ }^{139}$. This is the first time a vaccine has shown reduced rates of gonorrhoea in those inoculated, and follow-up studies are needed to determine whether the vaccine confers true protection or whether the reduced rates are not actually in response to the vaccination. If a viable vaccine were generated, producing and distributing a substantial amount of vaccine to reduce worldwide gonorrhoea prevalence would be a large economic challenge and unlikely to be undertaken by the pharmaceutical industry. The necessary follow-up studies, production and distribution of a viable vaccine will require non-profit and government support for funding.

\section{Antimicrobial resistance}

Without an effective vaccine, antibiotics have been the only effective method for controlling gonorrhoea, but the effectiveness of antibiotics is now in question. The main molecular mechanisms that are used by bacteria to develop antimicrobial resistance are protective alteration of antibiotic targets, decreased influx of antibiotics into the cell through transport proteins, increased efflux of antibiotics out of the cell via multidrug efflux pumps and expression of antibiotic-inactivating enzymes. Different strains of N. gonorrhoeae have evolved numerous resistance determinants using all of these mechanisms to inhibit killing by all major classes of antibiotics (FIG. 5a). There has been substantial research into $\beta$-lactam resistance mechanisms of $N$. gonorrhoeae. Transpeptidase penicillin-binding protein 2 ( $\mathrm{Pbp} 2$; encoded by the penA gene) is a periplasmic transpeptidase and the main lethal target of cephalosporins; most resistant isolates contain mosaic mutations in pen $A^{140}$. The pump

\section{Figure 5 Antibiotic resistance in Neisseria}

gonorrhoeae. a|Main resistance determinants of Neisseria gonorrhoeae. Transpeptidase penicillin-binding protein 2 (PBP2; encoded by penA) is a periplasmic transpeptidase and the main lethal target of cephalosporins; most resistant isolates contain mosaic mutations in penA. The efflux pump MtrC-MtrE-MtrD and its repressor MtrR contribute to N. gonorrhoeae resistance through antimicrobial efflux. The major porin protein, PorB, which is encoded by por $B$, is also a main resistance determinant that cannot manifest independently, but requires concomitant mutation in $m t r R$. $\mathbf{b} \mid$ Timeline of antibiotic-resistance development. Since the treatment of N. gonorrhoeae with sulfonamides in the 1930s, $N$. gonorrhoeae has acquired genetic resistance determinants that prevent killing by all major classes of antibiotics that are used as first-line methods of treatment for gonorrhoea ${ }^{143,145,147,181}$. As shown in the timeline, each new class of antibiotics that served as a first-line treatment for $\mathrm{N}$. gonorrhoeae has been stopped, as strains gained resistance. Recently, resistance was observed for the last available first-line treatment for N. gonorrhoeae infection, the extended-spectrum cephalosporins ${ }^{143}$. The ability of N. gonorrhoeae to evolve resistance has led the $\mathrm{WHO}$ and CDC to term it a 'superbug' and to speculate that if new therapies are not developed soon, we may face an era of untreatable antimicrobial-resistant gonorrhoea ${ }^{3}$.
MtrC-MtrD-MtrE and its repressor MtrR contribute to $N$. gonorrhoeae resistance through antimicrobial efflux $^{141}$. Variants of the major porin protein, which is encoded by por $B$, contribute to resistance to $\beta$-lactams, but resistance requires a concomitant mutation in $m t r R^{142}$. Interestingly, when all known resistance determinants are transformed into a susceptible recipient strain from a resistant strain, the transformants do not reconstitute the full level of resistance of the donor strain, suggesting the presence of one or more undiscovered factors that cannot easily be transferred to a recipient

\section{a}

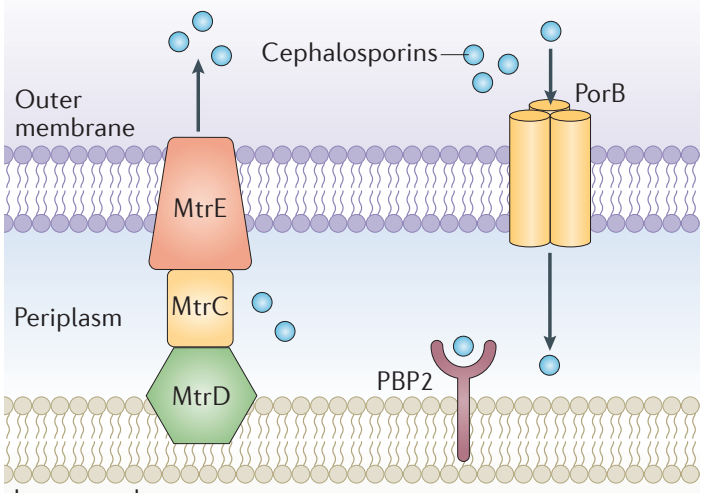

Inner membrane

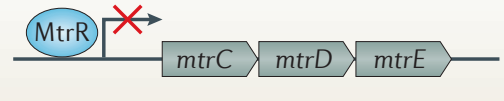

b

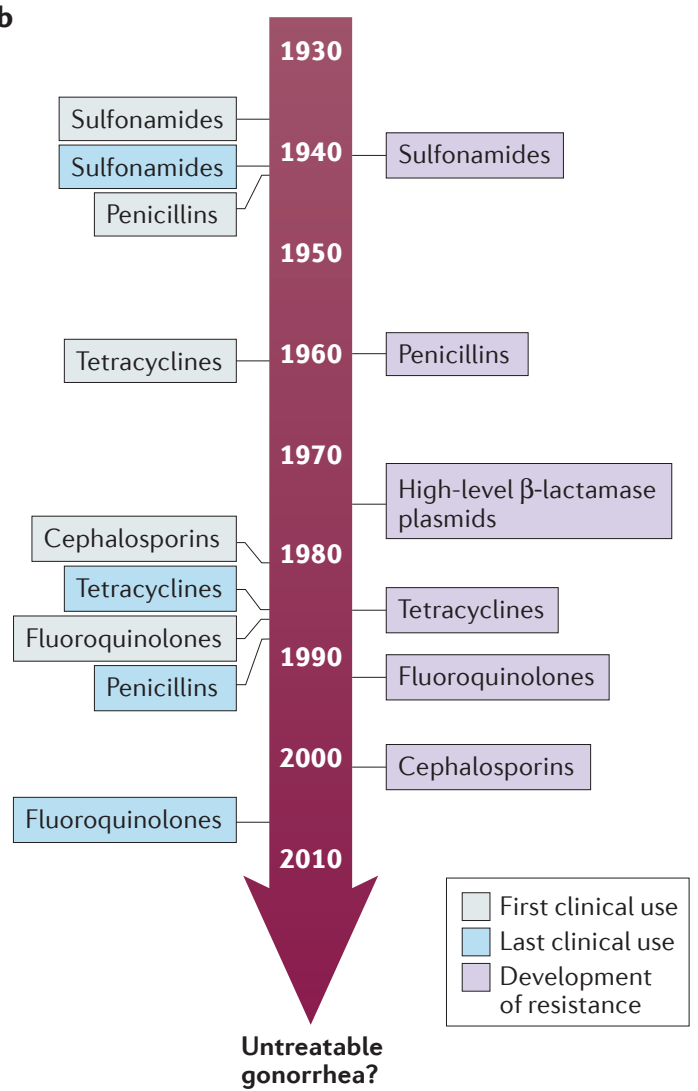


strain through transformation ${ }^{143}$. Moreover, expression levels of the Mtr efflux pump, controlled by MtrR, affect bacterial fitness during vaginal infection of female mice through an unknown mechanism ${ }^{144}$. This is an important example of how the development of antibiotic resistance can influence fitness, as, historically, the field has treated bacterial pathogenesis as a separate area of study from antimicrobial resistance. In addition, a greater understanding of $N$. gonorrhoeae antibiotic resistance determinants, especially in the context of increased fitness by enhancement of colonization or pathogenesis, is important because of the high potential for horizontal gene transfer between pathogenic and commensal Neisseria spp. within the human host, indicating a potential reservoir for antimicrobial-resistant genes.

The human history of antibiotic development is matched by the history of $N$. gonorrhoeae developing and retaining resistance to all new effective antibiotics (FIG. 5b). These antimicrobials include sulfonamides, penicillins, tetracyclines, macrolides and fluoroquinolones $^{143,145}$. The recent failures of treatments using cefixime and ceftriaxone, the extended-spectrum cephalosporins and $\beta$-lactam antibiotics that are used as the last available first-line treatments for gonorrhoea, have highlighted the potential for untreatable gonorrhoea to become a widespread public health epidemic ${ }^{146}$. Due to a lack of quality metadata on individuals who have been infected with $N$. gonorrhoeae, their sexual networks and the phenotypic and genotypic characteristics of the gonococcal population, we can only speculate on the reasons for antimicrobial resistance emergence and spread. Antimicrobial resistance has likely been facilitated by unrestricted access to and over-prescription of antimicrobials, particularly in the WHO's Western Pacific Region ${ }^{147}$. In addition, N. gonorrhoeae is naturally competent for transformation; thus, it is able to take up gonococcal DNA and to a lesser extent other bacterial DNA from the environment and recombine it efficiently with homologous sequences in the gonococcal genome ${ }^{148,149}$. The high propensity for N. gonorrhoeae to take up DNA from the environment adds to the likelihood that $N$. gonorrhoeae genes encoding antibiotic resistance determinants will mutate and become resistant. Transformation can produce mosaic alleles in genes that represent antimicrobial resistance determinants, wherein two orthologous or paralogous genes combine to form a mosaic, resistant variant gen $\mathrm{e}^{150}$. In addition, mutations can arise within a gene, conferring antibiotic resistance to that gene product. On the basis of previous observations, it is reasonable to predict that the spread of cephalosporin-resistant $N$. gonorrhoeae will continue to increase. Suspected multidrug-resistant strains are genotyped by multilocus sequence typing and $N$. gonorrhoeae multi-antigen sequence typing (NG-MAST), but it is important to continue hypothesis-driven molecular research to understand the molecular mechanisms of action for N. gonorrhoeae antibiotic resistance determinants. Indeed, this will enable the development of novel therapeutics and heighten our understanding of how resistance persists in a population and spreads among strains.

\section{Conclusions}

In this Review, we have summarized our knowledge of the course of $N$. gonorrhoeae pathogenesis from transmission, adherence, colonization and invasion to evasion of the innate and adaptive immune systems. We have emphasized the difficulty in studying a hostadapted human pathogen. Owing to the multifaceted nature of the urogenital tract, rectum and oropharynx (which are composed of many different types of epithelial tissue), the fact that innate immune cell composition varies by individual and the unknown concentrations of oxygen and nutrients, substantial challenges exist to develop tissue culture and animal models to study the obligate human pathogen N. gonorrhoeae. Advances in standardizing the cell culture techniques of primary tissue culture and transgenic mouse models may help to ameliorate these challenges. In addition, we have argued that asymptomatic infections are common in men and women. Though the prevailing dogma currently holds that infections in women are mainly asymptomatic whereas infections in men are not, many studies show asymptomatic infections are prevalent in both sexes. We argue that the prevailing hypothesis more likely stems from physiological and anatomical differences in the urogenital tract between sexes, making neutrophil influx much more obvious and gonorrhoea easier to diagnose in men than in women.

Owing to the host-restricted life cycle of N. gonorrhoeae and the limitations of existing tissue culture and animal model systems, many niches of the microenvironments that the gonococci inhabit and replicate within are unknown. Particularly, the nutrient and oxygen availability, the magnitude of innate immune responses and the microbiota composition (which are specific to the different sites within the male and female genital tracts) may be markedly different, requiring N. gonorrhoeae to adopt distinct adaptive programmes in response to local conditions as infection progresses. Deep sequencing of multiple clinical strains and further characterization of common intersecting gene regulons among strains can help elucidate which gene networks are important for colonization and pathogenesis. In addition, the continued development of tissue culture systems that are composed of different cell types - for example, modelling the different tissues that line the ascending vaginal, cervical and fallopian tube epithelia, and transgenic mouse models with humanized epithelial surface receptors - can help to generate more complex model microenvironments that are relevant to human infection. Very little data exist on the host and bacterial factors that contribute to infections that do not display overt symptoms, and more sensitive surveillance, screening and diagnostics are needed to characterize bacterial strains and host cell factors that contribute to asymptomatic colonization.

Despite the massive amount of data from sequencing projects showing the vast diversity of microbial species, scientists and policy makers still tend to think of all major groups of bacteria as being alike. N. gonorrhoeae is an example of a host-restricted organism that has been on a singular evolutionary path, existing 
currently (and likely throughout its evolution) as a commensal-like organism in equilibrium with the host but retaining the ability to elicit inflammation. Efficient horizontal gene transfer mechanisms have contributed to the rise in antimicrobial resistance. The stochastic alterations of gene expression and antigenic properties through phase variation and antigenic variation have hindered the development of viable vaccine candidates. Understanding the underlying mechanisms by which $N$. gonorrhoeae evades immune detection and develops antimicrobial resistance factors will aid in the development of new therapeutics for gonorrhoea.
1. World Health Organization. WHO Guidelines for the Treatment of Neisseria gonorrhoeae (WHO, 2016).

2. Carmona-Gutierrez, D., Kainz, K. \& Madeo, F. Sexually transmitted infections: old foes on the rise. Microb. Cell 3, 361-362 (2016).

3. Unemo, M. et al. The novel 2016 WHO Neisseria gonorrhoeae reference strains for global quality assurance of laboratory investigations: phenotypic, genetic and reference genome characterization. J. Antimicrob. Chemother. 71, 3096-3108 (2016).

4. Newman, L. et al. Global estimates of the prevalence and incidence of four curable sexually transmitted infections in 2012 based on systematic review and global reporting. PLOS ONE 10, e0143304 (2015).

5. Lee, J. S., Choi, H. Y., Lee, J. E., Lee, S. H. \& Oum, B. S Gonococcal keratoconjunctivitis in adults. Eye 16 646-649 (2002)

6. Noble, R. C., Cooper, R. M. \& Miller, B. R. Pharyngeal colonisation by Neisseria gonorrhoeae and Neisseria meningitidis in black and white patients attending a venereal disease clinic. Br. J. Vener. Dis. 55, 14-19 (1979).

7. Danby, C. S. et al. Patterns of extragenital chlamydia and gonorrhea in women and men who have sex with men reporting a history of receptive anal intercourse. Sex. Transm. Dis. 43, 105-109 (2016).

8. Little, J. W. Gonorrhea: update. Oral Surg., Oral Med Oral Pathol., Oral Radiol., Endodont. 101, 137-143 (2006).

9. Sandstrom, I. Etiology and diagnosis of neonatal conjunctivitis. Acta Paediatr. Scand. 76, 221-227 (1987).

10. Masi, A. T. \& Eisenstein, B. I. Disseminated gonococcal infection (DGI) and gonococcal arthritis (GCA): II. Clinical manifestations, diagnosis, complications, treatment, and prevention. Semin. Arthritis Rheum. 10, 173-197 (1981)

11. Hoffman, O. \& Weber, R. J. Pathophysiology and treatment of bacterial meningitis. Ther. Adv. Neurol. Disord. 2, 1-7 (2009).

12. Marri, P. R. et al. Genome sequencing reveals widespread virulence gene exchange among human Neisseria species. PLOS ONE 5, e11835 (2010).

13. Liu, G., Tang, C. M. \& Exley, R. M. Non-pathogenic Neisseria: members of an abundant, multi-habitat, diverse genus. Microbiology 161, 1297-1312 (2015).

14. Maiden, M. C. \& Harrison, O. B. Population and functional genomics of Neisseria revealed with gene-by-gene approaches. J. Clin. Microbiol. 54, 1949-1955 (2016).

15. Bratcher, H. B., Corton, C., Jolley, K. A., Parkhill, J. \& Maiden, M. C. A gene-by-gene population genomics platform: de novo assembly, annotation and genealogical analysis of 108 representative Neisseria meningitidis genomes. BMC Genomics 15, 1138 (2014).

16. Joseph, B. et al. Virulence evolution of the human pathogen Neisseria meningitidis by recombination in the core and accessory genome. PLOS ONE 6 e18441 (2011)

17. Maiden, M. C. Population genomics: diversity and virulence in the Neisseria. Curr. Opin. Microbiol. 11, 467-471 (2008)

18. Edwards, J. L. \& Apicella, M. A. The molecular mechanisms used by Neisseria gonorrhoeae to initiate infection differ between men and women. Clin. Microbiol. Rev. 17, 965-981 (2004).

19. Sparling, P. F. Biology of Neisseria gonorrhoeae. 3rd edn (McGraw-Hill, 1999).

20. Walker, C. K. \& Sweet, R. L. Gonorrhea infection in women: prevalence, effects, screening, and management. Int. J. Women' Health 3, 197-206 (2011).

21. Jordan, S. J., Schwebke, J. R., Aaron, K. J., Van Der Pol, B. \& Hook, E. W. 3rd. Meatal swabs contain less cellular material and are associated with a decrease in Gram stain smear quality compared to urethral swabs in men. J. Clin. Microbiol. 55, 2249-2254 (2017).

22. Muzny, C. A. et al. Sexually transmitted infection risk among women is not fully explained by partner numbers. South Med. J. 110, 161-167 (2017)

23. Grimley, D. M. et al. Sexually transmitted infections among urban shelter clients. Sex. Transm. Dis. 33 666-669 (2006)

24. Geisler, W. M., Yu, S. \& Hook, E. W. 3rd. Chlamydial and gonococcal infection in men without polymorphonuclear leukocytes on gram stain: implications for diagnostic approach and management. Sex. Transm. Dis. 32, 630-634 (2005).

25. Xiong, M. et al. Analysis of the sex ratio of reported gonorrhoea incidence in Shenzhen, China. BMJ Open 6, e009629 (2016).

This epidemiological study of 1,106 male and 1,420 female participants in Shenzhen, China, shows that undiagnosed, unreported gonorrhoea infections were common in both men and women and that the reported incidence sex ratio was overestimated by a factor of 7.9 .

26. Hook, E. W. 3rd. Gender differences in risk for sexually transmitted diseases. Am. J. Med. Sci. 343, 10-11 (2012).

27. Hedges, S. R. et al. Limited local and systemic antibody responses to Neisseria gonorrhoeae during uncomplicated genital infections. Infect. Immun. 67 , 3937-3946 (1999)

28. Fichorova, R. N., Desai, P. J., Gibson, F. C. 3rd \& Genco, C. A. Distinct proinflammatory host responses to Neisseria gonorrhoeae infection in immortalized human cervical and vaginal epithelial cells. Infect. Immun. 69, 5840-5848 (2001).

29. Papp, J. R., Schachter, J., Gaydos, C. A. \& Van Der Pol, B. Recommendations for the laboratory based detection of Chlamydia trachomatis and Neisseria gonorrhoeae - 2014. MMWR Morb. Mortal. Wkly Rep. 63, 1-19 (2014).

30. James-Holmquest, A. N., Swanson, J., Buchanan, T. M., Wende, R. D. \& Williams, R. P. Differential attachment by piliated and nonpiliated Neisseria gonorrhoeae to human sperm. Infect. Immun. 9, 897-902 (1974).

31. Harvey, H. A. et al. Gonococcal lipooligosaccharide is a ligand for the asialoglycoprotein receptor on human sperm. Mol. Microbiol. 36, 1059-1070 (2000). This study shows that gonococcal LOS binds to asialoglycoprotein receptor 1 (ASGPR1) on human sperm, possibly contributing to male-to-female transmission.

32. Cohen, M. S. et al. Human experimentation with Neisseria gonorrhoeae: rationale, methods, and implications for the biology of infection and vaccine development. J. Infect. Dis. 169, 532-537 (1994).

33. Ketterer, M. R. et al. Desialylation of Neisseria gonorrhoeae Lipooligosaccharide by Cervicovaginal Microbiome Sialidases: The Potential for Enhancing Infectivity in Men. J. Infect. Dis. 214, 1621-1628 (2016)

34. Higashi, D. L. et al. Dynamics of Neisseria gonorrhoeae attachment: microcolony development, cortical plaque formation, and cytoprotection. Infect Immun. 75, 4743-4753 (2007).

35. Craig, L., Pique, M. E. \& Tainer, J. A. Type IV pilus structure and bacterial pathogenicity. Nat. Rev. Microbiol. 2, 363-378 (2004).

36. Obergfell, K. P. \& Seifert, H. S. The pilin N-terminal domain maintains Neisseria gonorrhoeae transformation competence during pilus phase variation. PLOS Genet. 12, e1006069 (2016).

37. Berry, J.-L. \& Pelicic, V. Exceptionally widespread nanomachines composed of type IV pilins: the prokaryotic Swiss Army knives. FEMS Microbiol. Rev. 39, 134-154 (2015).

38. Cahoon, L. A. \& Seifert, H. S. Transcription of a cisacting, noncoding, small RNA is required for pilin antigenic variation in Neisseria gonorrhoeae. PLOS Pathog. 9, e1003074 (2013).

This study demonstrates that transcription of a small, cis-acting, non-coding RNA initiates within the guanine quartet (G4) coding sequence enables the formation of the G4 structure required for pilin antigenic variation.

39. Dietrich, M. et al. Activation of NF-kappaB by Neisseria gonorrhoeae is associated with microcolony formation and type IV pilus retraction. Cell. Microbiol. 13, 1168-1182 (2011)

40. Swanson, J., Barrera, O., Sola, J. \& Boslego, J. Expression of outer membrane protein II by gonococci in experimental gonorrhea. J. Exp. Med. 168 2121-2129 (1988)

41. Jerse, A. E. et al. Multiple gonococcal opacity proteins are expressed during experimental urethral infection in the male. J. Exp. Med. 179, 911-920 (1994). This study shows that when Opa-less variants of $N$. gonorrhoeae strain FA1090 were inoculated into human male volunteers, a majority of bacteria cultured from the infected subjects were Opa-positive and expressed a variety of Opa variants.

42. Virji, M., Makepeace, K., Ferguson, D. J. \& Watt, S. M. Carcinoembryonic antigens (CD66) on epithelial cells and neutrophils are receptors for Opa proteins of pathogenic neisseriae. Mol. Microbiol. 22, 941-950 (1996).

43. Simms, A. N. \& Jerse, A. E. In vivo selection for Neisseria gonorrhoeae opacity protein expression in the absence of human carcinoembryonic antigen cell adhesion molecules. Infect. Immun. 74, 2965-2974 (2006).

44. Lambden, P. R., Heckels, J. E., James, L. T. \& Watt, P. J. Variations in surface protein composition associated with virulence properties in opacity types of Neisseria gonorrhoeae. J. Gen. Microbiol. 114, 305-312 (1979).

45. Stern, A., Brown, M., Nickel, P. \& Meyer, T. F. Opacity genes in Neisseria gonorrhoeae: control of phase and antigenic variation. Cell 47, 61-71 (1986).

46. Swanson, J. et al. Gonococcal pilin variants in experimental gonorrhea. J. Exp. Med. 165, 1344-1357 (1987)

47. James, J. F. \& Swanson, J. Studies on gonococcus infection. XIII. Occurrence of color/opacity colonial variants in clinical cultures. Infect. Immun. 19 332-340 (1978)

48. Seifert, H. S., Wright, C. J., Jerse, A. E., Cohen, M. S \& Cannon, J. G. Multiple gonococcal pilin antigenic variants are produced during experimental human infections. J. Clin. Invest. 93, 2744-2749 (1994).

49. Anderson, M. T., Byerly, L., Apicella, M. A. \& Seifert, H. S. Seminal plasma promotes Neisseria gonorrhoeae aggregation and biofilm formation. J. Bacteriol. 198, 2228-2235 (2016).

50. Steichen, C. T., Cho, C., Shao, J. Q. \& Apicella, M. A The Neisseria gonorrhoeae biofilm matrix contains DNA, and an endogenous nuclease controls its incorporation. Infect. Immun. 79, 1504-1511 (2011).

51. Greiner, L. L. et al. Biofilm Formation by Neisseria gonorrhoeae. Infect. Immun. 73, 1964-1970 (2005).

52. Steichen, C. T., Shao, J. Q., Ketterer, M. R. \& Apicella, M. A. Gonococcal cervicitis: a role for biofilm in pathogenesis. J. Infect. Dis. 198, 1856-1861 (2008).

53. Wetzler, L. M., Blake, M. S., Barry, K. \& Gotschlich, E. C. Gonococcal porin vaccine evaluation: comparison of Por proteosomes, liposomes, and blebs isolated from $\mathrm{rmp}$ deletion mutants. J. Infect. Dis. 166, 551-555 (1992).

54. Song, W., Ma, L., Chen, R. \& Stein, D. C. Role of lipooligosaccharide in Opa-independent invasion of Neisseria gonorrhoeae into human epithelial cells. J. Exp. Med. 191, 949-960 (2000)

55. van Vliet, S. J. et al. Variation of Neisseria gonorrhoeae lipooligosaccharide directs dendritic cell-induced T helper responses. PLOS Pathog. 5, e1000625 (2009). 
56. Wetzler, L. M., Barry, K., Blake, M. S. \& Gotschlich, E. C. Gonococcal lipooligosaccharide sialylation prevents complement-dependent killing by immune sera. Infect. Immun. 60, 39-43 (1992). This study shows that sialylation of gonococcal LOS prevents opsonophagocytosis by immune sera, which led to the later confirmation that sialylation of LOS prevents complement activation and killing

57. Kellogg, D. S. et al. Neisseria gonorrhoeae. I. Virulence genetically linked to clonial variation. J. Bacteriol. 85, 1274-1279 (1963).

58. Spence, J. M., Wright, L. \& Clark, V. L. Laboratory maintenance of Neisseria gonorrhoeae. Curr. Protoc. Microbiol., Unit 4A (2008).

This study compares selectively passaged, piliated $N$. gonorrhoeae capable of infecting human volunteers with non-selectively passaged, non-piliated clonal variants that became non-infectious, enabling researchers to realize that infectivity can be phenotypically followed by observing piliated and non-piliated colony morphology.

59. Platt, D. J. Carbon dioxide requirement of Neisseria gonorrhoeae growing on a solid medium. J. Clin. Microbiol. 4, 129-132 (1976).

60. St Amant, D. C. Valentin-Bon, I. E. \& Jerse, A. E. Inhibition of Neisseria gonorrhoeae by Lactobacillus species that are commonly isolated from the female genital tract. Infect. Immun. 70, 7169-7171 (2002)

61. Spurbeck, R. R. \& Arvidson, C. G. Inhibition of Neisseria gonorrhoeae epithelial cell interactions by vaginal Lactobacillus species. Infect. Immun. 76, 3124-3130 (2008).

62. Spurbeck, R. R. \& Arvidson, C. G. Lactobacillus jensenii surface-associated proteins inhibit Neisseria gonorrhoeae adherence to epithelial cells. Infect. Immun. 78, 3103-3111 (2010).

63. Cassat, J. E. \& Skaar, E. P. Iron in infection and immunity. Cell Host Microbe 13, 509-519 (2013).

64. Doherty, C. P. Host-pathogen interactions: the role of iron. J. Nutr. 137, 1341-1344 (2007).

65. Bonnah, R. A. \& Schryvers, A. B. Preparation and characterization of Neisseria meningitidis mutants deficient in production of the human lactoferrinbinding proteins LbpA and LbpB. J. Bacteriol. 180 , 3080-3090 (1998)

66. Noinaj, N., Buchanan, S. K. \& Cornelissen, C. N. The transferrin-iron import system from pathogenic Neisseria species. Mol. Microbiol. 86, 246-257 (2012).

67. Evans, R. W. \& Oakhill, J. S. Transferrin-mediated iron acquisition by pathogenic Neisseria. Biochem. Soc. Trans. 30, 705-707 (2002)

68. Kehl-Fie, T. E. \& Skaar, E. P. Nutritional immunity beyond iron: a role for manganese and zinc. Curr. Opin. Chem. Biol. 14, 218-224 (2010).

69. Ovcinnikov, N. M. \& Delektorskij, V. V. Electron microscope studies of gonococci in the urethral secretions of patients with gonorrhoea. Br. J. Vener. Dis. 47, 419-439 (1971).

70. Farzadegan, H. \& Roth, I. L. Scanning electron microscopy and freeze-etching of gonorrhoeal urethra exudate. Br. J. Vener. Dis. 51, 83-91 (1975)

71. Evans, B. A. Ultrastructural study of cervical gonorrhea. J. Infect. Dis. 136, 248-255 (1977).

72. King, G., James, J. F. \& Swanson, J. Studies on gonococcus infection. XI. Comparison of in vivo and vitro association of Neisseria gonorrhoeae with human neutrophils. J. Infect. Dis. 137, 38-43 (1978)

73. Apicella, M. A. et al. The pathogenesis of gonococcal urethritis in men: confocal and immunoelectron microscopic analysis of urethral exudates from men infected with Neisseria gonorrhoeae. J. Infect. Dis. 173, 636-646 (1996).

74. Criss, A. K. \& Seifert, H. S. A bacterial siren song: intimate interactions between Neisseria and neutrophils. Nat. Rev. Microbiol. 10, 178-190 (2012).

75. Lucas, C. E., Hagman, K. E., Levin, J. C., Stein, D. C. \& Shafer, W. M. Importance of lipooligosaccharide structure in determining gonococcal resistance to hydrophobic antimicrobial agents resulting from the mtr efflux system. Mol. Microbiol. 16, 1001-1009 (1995).

76. Zalucki, Y. M., Dhulipala, V. \& Shafer, W. M. Dueling regulatory properties of a transcriptional activator (MtrA) and repressor (MtrR) that control efflux pump gene expression in Neisseria gonorrhoeae. mBio 3 e00446-12 (2012)

This study compares the binding affinities and regulatory competition between MtrC-MtrD-MtrE efflux pump operon activator MtrA and repressor
MtrR, building on previous data characterizing this important antimicrobial resistance pump and its transcriptional regulation

77. Thaler, C. J., Vanderpuye, O. A., McIntyre, J. A. \& Faulk, W. P. Lactoferrin binding molecules in human seminal plasma. Biol Reprod 43, 712-717 (1990).

78. Mercante, A. D. et al. MpeR regulates the mtr efflux locus in Neisseria gonorrhoeae and modulates antimicrobial resistance by an iron-responsive mechanism. Antimicrob. Agents Chemother 56, 1491-1501 (2012)

79. Laskos, L., Ryan, C. S., Fyfe, J. A. \& Davies, J. K. The RpoH-mediated stress response in Neisseria gonorrhoeae is regulated at the level of activity. J. Bacteriol. 186, 8443-8452 (2004).

80. Householder, T. C., Belli, W. A., Lissenden, S. Cole, J. A. \& Clark, V. L. cis- and trans- acting elements involved in the regulation of aniA, the gene encoding the major anaerobically induced outer membrane protein in Nesseria gonorrhoeae. J. Bacteriol. 181 5411-5551 (1999).

81. Mellies, J., Rudel, T. \& Meyer, T. F. Transcriptional regulation of pilC2 in Neisseria gonorrhoeae: response to oxygen availability and evidence for growth-phase regulation in Escherichia coli. Mol. Gen. Genet. 255, 285-293 (1997).

82. Whitehead, R. N. et al. The small FNR regulon of Neisseria gonorrhoeae: comparison with the larger Escherichia coli FNR regulon and interaction with the NarQ-NarP regulon. BMC Genomics 8, 35 (2007).

83. Berish, S. A., Subbarao, S., Chen, C. Y., Trees, D. L. $£$ Morse, S. A. Identification and cloning of a fur homolog from Neisseria gonorrhoeae. Infect. Immun 61, 4599-4606 (1993)

This study identifies and initially characterizes the major iron-regulatory protein Fur in N. gonorrhoeae.

84. Isabella, V. M. \& Clark, V. L. Deep sequencing-based analysis of the anaerobic stimulon in Neisseria gonorrhoeae. BMC Genomics 12, 51 (2011). This study identifies a wide array of genes that are differentially expressed under aerobic and anaerobic conditions in microaerophile N. gonorrhoeae, highlighting the large overlap among genes that are differentially regulated in response to low oxygen, changes in iron levels and the presence of reactive oxygen species.

85. Ducey, T. F., Carson, M. B., Joshua, O. \& Stintzi, A. P. $\&$ Dyer, D. W. Identification of the iron-responsive genes of Neisseria gonorrhoaea by microarray analysis in defined medium. J. Bacteriol. 187 4865-4874 (2005)

86. Stohl, E. A., Criss, A. K. \& Seifert, H. S. The transcriptome response of Neisseria gonorrhoeae to hydrogen peroxide reveals genes with previously uncharacterized roles in oxidative damage protection. Mol. Microbiol. 58, 520-532 (2005).

87. Makino, S., van Putten, J. P. \& Meyer, T. F. Phase variation of the opacity outer membrane protein controls invasion by Neisseria gonorrhoeae into human epithelial cells. EMBO J. 10, 1307-1315 (1991)

This study shows a positive correlation between the expression of Opa proteins and the binding and invasion of $N$. gonorrhoeae into Chang conjunctiva human epithelial cells.

88. Snyder, L. A., Butcher, S. A. \& Saunders, N. J. Comparative whole-genome analyses reveal over 100 putative phase-variable genes in the pathogenic Neisseria spp. Microbiology 147, 2321-2332 (2001)

89. Jordan, P. W., Snyder, L. A. \& Saunders, N. J. Strain-specific differences in Neisseria gonorrhoeae associated with the phase variable gene repertoire. BMC Microbiol. 5, 21 (2005).

90. Srikhanta, Y. N. et al. Phasevarions mediate random switching of gene expression in pathogenic Neisseria. PLOS Pathog. 5, e 1000400 (2009).

This study characterizes phase-variable DNA methyltransferase activity in $N$. gonorrhoeae, showing that it affects the expression of virulence-related genes, antimicrobial resistance, human epithelial cervical cell interactions and biofilm formation.

91. Cawthorne J A Beatson S. A Srikhanta, Y N Fox, K. L. \& Jennings, M. P. Origin of the diversity in DNA recognition domains in phasevarion associated modA genes of pathogenic Neisseria and Haemophilus influenzae. PLOS ONE 7, e32337 (2012).

92. Jen, F. E., Seib, K. L. \& Jennings, M. P. Phasevarions mediate epigenetic regulation of antimicrobial susceptibility in Neisseria meningitidis. Antimicrob. Agents Chemother. 58, 4219-4221 (2014).

93. Post, D. M. B. et al. Identification and characterization of AckA-dependent protein acetylation in Neisseria gonorrhoeae. PLOS ONE 12, e0179621 (2017).

94. Seib, K. L., Jen, F. E., Scott, A. L., Tan, A. \& Jennings, M. P. Phase variation of DNA methyltransferases and the regulation of virulence and immune evasion in the pathogenic Neisseria. Pathog. Dis. 75, ftx080 (2017)

95. Gibson, F. P., Leach, D. R. \& Lloyd, R. G. Identification of $s b c D$ mutations as cosuppressors of recBC that allow propagation of DNA palindromes in Escherichia coli K-12. J. Bacteriol. 174, 1222-1228 (1992).

96. Jackson, L. A., Pan, J. C., Day, M. W. \& Dyer, D. W. Control of RNA stability by NrrF, an iron-regulated small RNA in Neisseria gonorrhoeae. J. Bacteriol. 195, 5166-5173 (2013).

97. Ngampasutadol, J. et al. Human factor $\mathrm{H}$ interacts selectively with Neisseria gonorrhoeae and results in species-specific complement evasion. J. Immunol. 180, 3426-3435 (2008) This study demonstrates how sialylated LOS binds human factor $\mathrm{H}$ and prevents complement-mediated killing of $N$. gonorrhoeae.

98. Densen, P. Interaction of complement with Neisseria meningitidis and Neisseria gonorrhoeae. [Review] Clin. Microbiol. Rev. 2 (Suppl.), S11-S1 7 (1989).

99. Petersen, B. H., Graham, J. A. \& Brooks, G. F. Human deficiency of the eighth component of complement. The requirement of $\mathrm{C} 8$ for serum Neisseria gonorrhoeae bactericidal activity. J. Clin. Invest. $\mathbf{5 7}$ 283-290 (1976)

100. Edwards, J. L., Brown, E. J., Ault, K. A. \& Apicella, M. A. The role of complement receptor 3 (CR3) in Neisseria gonorrhoeae infection of human cervical epithelia. Cell. Microbiol. 3, 611-622 (2001).

101. Edwards, J. L. \& Apicella, M. A. The role of lipooligosaccharide in Neisseria gonorrhoeae pathogenesis of cervical epithelia: lipid A serves as a C3 acceptor molecule. Cell. Microbiol. 4, 585-598 (2002).

102. Edwards, J. L. et al. A co-operative interaction between Neisseria gonorrhoeae and complement receptor 3 mediates infection of primary cervical epithelial cells. Cell. Microbiol. 4, 571-584 (2002).

103. Schweinle, J. E. et al. Interaction of Neisseria gonorrhoeae with classical complement components, C1-inhibitor, and a monoclonal antibody directed against the Neisserial H.8 antigen. J. Clin. Invest. $\mathbf{8 3}$, 397-403 (1989).

104. Ram, S. et al. A novel sialic acid binding site on factor $\mathrm{H}$ mediates serum resistance of sialylated Neisseria gonorrhoeae. J. Exp. Med. 187, 743-752 (1998).

105. Ram, S. et al. Binding of complement factor $\mathrm{H}$ to loop 5 of porin protein 1A: a molecular mechanism of serum resistance of nonsialylated Neisseria gonorrhoeae. J. Exp. Med. 188, 671-680 (1998).

106. Ram, S et al. Binding of C4b-binding protein to porin a molecular mechanism of serum resistance of Neisseria gonorrhoeae. J. Exp. Med. 193, 281-295 (2001).

107. Gill, D. B. \& Atkinson, J. P. CD46 in Neisseria pathogenesis. Trends Mol Med 10, 459-465 (2004).

108. Feinen, B. \& Russell, M. W. Contrasting roles of IL-22 and IL-1 7 in murine genital tract infection by Neisseria gonorrhoeae. Front. Immunol. 3, 11 (2012).

109. Edwards, J. L. \& Butler, E. K. The pathobiology of Neisseria gonorrhoeae lower female genital tract infection. Front. Microbiol. 2, 102 (2011).

110. Melly, M. A., McGee, Z. A. \& Rosenthal, R. S. Ability of monomeric peptidoglycan fragments from Neisseria gonorrhoeae to damage human fallopian-tube mucosa. J. Infect. Dis. 149, 378-386 (1984). This study demonstrates the ability of differen $N$. gonorrhoeae peptidoglycan monomers to damage human fallopian tube mucosal cells in tissue culture.

111. Mavrogiorgos, N., Mekasha, S., Yang, Y., Kelliher, M. A. \& Ingalls, R. R. Activation of NOD receptors by Neisseria gonorrhoeae modulates the innate immune response. Innate Immun. 20 377-389 (2014)

112. Fisette, P. L., Ram, S., Andersen, J. M., Guo, W. \& Ingalls, R. R. The Lip lipoprotein from Neisseria gonorrhoeae stimulates cytokine release and NF-kappaB activation in epithelial cells in a Toll-like receptor 2-dependent manner. J. Biol. Chem. 278 46252-46260 (2003). 
113. Massari, P. et al. Cutting edge: Immune stimulation by neisserial porins is toll-like receptor 2 and MyD88 dependent. J. Immunol. 168, 1533-1537 (2002).

114. Chateau, A. \& Seifert, H. S. Neisseria gonorrhoeae survives within and modulates apoptosis and inflammatory cytokine production of human macrophages. Cell. Microbiol. 18, 546-560 (2016).

115. Gaudet, R. G. et al. Cytosolic detection of the bacterial metabolite HBP activates TIFA-dependent innate immunity. Science 348, 1251-1255 (2015).

116. Ortiz, M. C. et al. Neisseria gonorrhoeae modulates immunity by polarizing human macrophages to a M2 profile. PLOS ONE 10, e0130713 (2015).

117. Sadarangani, M., Pollard, A. J. \& Gray-Owen, S. D. Opa proteins and CEACAMs: pathways of immune engagement for pathogenic Neisseria. FEMS Microbiol. Rev. 35, 498-514 (2011).

118. Schmitter, T., Agerer, F., Peterson, L., Munzner, P. \& Hauck, C. R. Granulocyte CEACAM3 is a phagocytic receptor of the innate immune system that mediates recognition and elimination of human-specific pathogens. J. Exp. Med. 199, 35-46 (2004).

119. Sarantis, H. \& Gray-Owen, S. D. The specific innate immune receptor CEACAM3 triggers neutrophil bactericidal activities via a Syk kinase-dependent pathway. Cell. Microbiol. 9, 2167-2180 (2007).

120. Packiam, M., Veit, S. J., Anderson, D. J., Ingalls, R. R. \& Jerse, A. E. Mouse strain-dependent differences in susceptibility to Neisseria gonorrhoeae infection and induction of innate immune responses. Infect. Immun. 78, 433-440 (2010)

121. Dilworth, J. A., Hendley, J. O. \& Mandell, G. L. Attachment and ingestion of gonococci human neutrophils. Infect. Immun. 11, 512-516 (1975). This early study shows adherence and ingestion of two different gonococci strains by polymorphonuclear leukocyte neutrophils (PMNs).

122. Criss, A. K. \& Seifert, H. S. Neisseria gonorrhoeae suppresses the oxidative burst of human polymorphonuclear leukocytes. Cell. Microbiol. 10 2257-2270 (2008).

This study demonstrates how different types of Opa-expressing or Opa-less $\boldsymbol{N}$. gonorrhoeae grown under different conditions differ in their ability to elicit a PMN oxidative burst, as well as the ability of some strains to inhibit the PMN oxidative burst.

123. Gunderson, C. W. \& Seifert, H. S. Neisseria gonorrhoeae elicits extracellular traps in primary neutrophil culture while suppressing the oxidative burst. mBio 6, e02452-14 (2015).

124. Criss, A. K., Katz, B. Z. \& Seifert, H. S. Resistance of Neisseria gonorrhoeae to non-oxidative killing by adherent human polymorphonuclear leucocytes. Cell. Microbiol. 11, 1074-1087 (2009).

125. Johnson, M. B. \& Criss, A. K. Resistance of Neisseria gonorrhoeae to neutrophils. Front. Microbiol. 2, 77 (2011).

126. Soler-Garcia, A. A. \& Jerse, A. E. A. Neisseria gonorrhoeae catalase mutant is more sensitive to hydrogen peroxide and paraquat, an inducer of toxic oxygen radicals. Microb. Pathog. 37, 55-63 (2004).

127. Gunesekere, I. C. et al. Ecf, an alternative sigma facto from Neisseria gonorrhoeae, controls expression of msrAB, which encodes methionine sulfoxide reductase. J. Bacteriol. 188, 3463-3469 (2006).

128. Pilch, B. \& Mann, M. Large-scale and high-confidence proteomic analysis of human seminal plasma. Genome Biol. 7, R40 (2006).

129. Schmidt, K. A. et al. Experimental gonococcal urethritis and reinfection with homologous gonococci in male volunteers. Sex. Transm. Dis. 28, 555-564 (2001).

130. Cahoon, L. A. \& Seifert, H. S. Focusing homologous recombination: pilin antigenic variation in the pathogenic Neisseria. Mol. Microbiol. 81, 1136-1143 (2011).

131. Mandrell, R. E., Griffiss, J. M. \& Macher, B. A Lipooligosaccharides (LOS) of Neisseria gonorrhoeae and Neisseria meningitidis have components that are immunochemically similar to precursors of human blood group antigens. Carbohydrate sequence specificity of the mouse monoclonal antibodies that recognize crossreacting antigens on LOS and human erythrocytes [published erratum appears in J. Exp Med 1988 Oct $1 ; 168,1517]$. J. Exp. Med. 168 107-126 (1988)

132. Mandrell, R. E. Further antigenic similarities of Neisseria gonorrhoeae lipooligosaccharides and human glycosphingolipids. Infect. Immun. 60 ,

3017-3020 (1992)

133. Gulati, S., McQuillen, D. P., Mandrell, R. E., Jani, D. B. \& Rice, P. A. Immunogenicity of Neisseria gonorrhoeae lipooligosaccharide epitope $2 \mathrm{C} 7$, widely expressed in vivo with no immunochemical similarity to human glycosphingolipids. J. Infect. Dis. 174, 1223-1237 (1996).

134. Liu, Y., Islam, E., Jarvis, G., Gray-Owen, S. \& Russell, M. Neisseria gonorrhoeae selectively suppresses the development of Th1 and Th2 cells, and enhances Th1 7 cell responses, through TGF- $\alpha-$ dependent mechanisms. Mucosal Immunol. $\mathbf{5}$ 320-331 (2012).

135. Liu, Y., Liu, W. \& Russell, M. W. Suppression of host adaptive immune responses by Neisseria gonorrhoeae: role of interleukin 10 and type 1 regulatory T cells. Mucosal Immunol. 7, 165-176 (2014).

136. Zhu, W. et al. Neisseria gonorrhoeae suppresses dendritic cell-induced, antigen-dependent CD4 T cell proliferation. PLOS ONE 7, e41260 (2012).

137. Liu, Y., Feinen, B. \& Russell, M. W. New concepts in immunity to Neisseria gonorrhoeae: innate responses and suppression of adaptive immunity favor the pathogen, not the host. Front. Microbiol. 2, 52 (2011).

138. Jerse, A. E., Bash, M. C. \& Russell, M. W. Vaccines against gonorrhea: current status and future challenges. Vaccine 32, 1579-1587 (2014).

139. Petousis-Harris, H. et al. Effectiveness of a group B outer membrane vesicle meningococcal vaccine against gonorrhoea in New Zealand: a retrospective case-control study. Lancet 390, 1603-1610 (2017).

140. Zhao, S. et al. Genetics of chromosomally mediated intermediate resistance to ceftriaxone and cefixime in Neisseria gonorrhoeae. Antimicrob. Agents Chemother. 53, 3744-3751 (2009).

141. Hagman, K. E. et al. Resistance of Neisseria gonorrhoeae to antimicrobial hydrophobic agents is modulated by the mtrRCDE efflux system. Microbiology 141, 611-622 (1995)

142. Zhao, S., Tobiason, D. M., Hu, M., Seifert, H. S. \& Nicholas, R. A. The penC mutation conferring antibiotic resistance in Neisseria gonorrhoeae arises from a mutation in the PilQ secretin that interferes with multimer stability. Mol. Microbiol. $\mathbf{5 7}$ 1238-1251 (2005)

143. Unemo, M. \& Shafer W. M. Antimicrobial resistance in Neisseria gonorrhoeae in the 21 st century: past, evolution, and future. Clin. Microbiol. Rev. 27, 587-613 (2014).

144. Jerse, A. E. et al. A gonococcal efflux pump system enhances bacterial survival in a female mouse model of genital tract infection. Infect. Immun. 71 5576-5582 (2003)

145. Unemo, M. \& Shafer, W. M. Antibiotic resistance in Neisseria gonorrhoeae: origin, evolution, and lessons learned for the future. Ann. NY Acad. Sci. 1230 E19-E28 (2011).

146. Hook, M. W., Schafer, W., Deal, C., Kirkcaldy, R. D. \& Iskander, J. CDC Grand Rounds: the growing threat of multidrug-resistant gonorrhea. MMWR Morb. Mortal. Wkly Rep. 62, 103-106 (2013).

147. Unemo, M. \& Nicholas, R. A. Emergence of multidrugresistant, extensively drug-resistant and untreatable gonorrhea. Future Microbiol. 7, 1401-1422 (2012).

148. Aas, F. E., Lovold, C. \& Koomey, M. An inhibitor of DNA binding and uptake events dictates the proficiency of genetic transformation in Neisseria gonorrhoeae: mechanism of action and links to Type IV pilus expression. Mol. Microbiol. 46, 1441-1450 (2002).

149. Hamilton, H. L. \& Dillard, J. P. Natural transformation of Neisseria gonorrhoeae: from DNA donation to homologous recombination. Mol. Microbiol. 59 376-385 (2006).

150. Bowler, L. D., Zhang, Q. Y., Riou, J. Y. \& Spratt, B. G. Interspecies recombination between the penA genes of Neisseria meningitidis and commensal Neisseria species during the emergence of penicillin resistance in $N$. meningitidis: natural events and laboratory simulation. J. Bacteriol. 176, 333-337 (1994).

151. Ng, L. K. \& Martin, I. E. The laboratory diagnosis of Neisseria gonorrhoeae. Can. J. Infect. Dis. Med. Microbiol. 16, 15-25 (2005)

152. De Silva, D. et al. Whole-genome sequencing to determine transmission of Neisseria gonorrhoeae: an observational study. Lancet Infect. Dis. 16

1295-1303 (2016).

153. Harrison, O. B. et al. Genomic analysis of urogenital and rectal Neisseria meningitidis isolates reveals encapsulated hyperinvasive meningococci and coincident multidrug-resistant gonococci. Sex. Transm. Infect. 93, 445-451 (2017).

154. Johnson, L. F. \& Lewis, D. A. The effect of genital tract infections on HIV-1 shedding in the genital tract: a systematic review and meta-analysis. Sex. Transm. Dis. 35, 946-959 (2008).

155. Kalichman, S. C., Pellowski, J. \& Turner, C. Prevalence of sexually transmitted co-infections in people living with HIV/AIDS: systematic review with implications for using HIV treatments for prevention. Sex. Transm. Infect. 87, 183-190 (2011).

156. Jerse, A. E. et al. Estradiol-Treated female mice as surrogate hosts for Neisseria gonorrhoeae genita tract infections. Front. Microbiol. 2, 107 (2011).

157. Zarantonelli, M. L. et al. Transgenic mice expressing human transferrin as a model for meningococcal infection. Infect. Immun. 75, 5609-5614 (2007).

158. Gu, A., Zhang, Z., Zhang, N., Tsark, W. \& Shively, J. E. Generation of human CEACAM1 transgenic mice and binding of Neisseria Opa protein to their neutrophils. PLOS ONE 5, e 10067 (2010).

159. Li, G. et al. Establishment of a human CEACAM 1 transgenic mouse model for the study of gonococcal infections. J. Microbiol. Methods 87, 350-354 (2011).

This study presents and characterizes a transgenic mouse model for gonorrhoea infection wherein the mouse has been made to express a humanized CEACAM receptor molecule important for adherence and colonization, enabling $N$. gonorrhoeae to intravaginally colonize the mouse.

160. Winther-Larsen, H. C. et al. in 13th International Pathogenic Neisseria Conference (eds Caugant, D. A. \& Wedege, E.) 37 (Oslo, 2002)

161. Pearce, W. A. \& Buchanan, T. M. Attachment role of gonococcal pili. Optimum conditions and quantitation of adherence of isolated pili to human cells in vitro. J. Clin. Invest. 61, 931-943 (1978)

162. Kaparakis, M. et al. Bacterial membrane vesicles deliver peptidoglycan to NOD1 in epithelial cells. Cell. Microbiol. 12, 372-385 (2010)

163. Zhou, X. et al. Hexa-acylated lipid A is required for host inflammatory response to Neisseria gonorrhoeae in experimental gonorrhea. Infect. Immun. 82, 184-192 (2014)

164. Singleton, T. E., Massari, P. \& Wetzler, L. M. Neisserial porin-induced dendritic cell activation is MyD88 and TLR2 dependent. J. Immunol. 174, 3545-3550 (2005).

165. Liu, X. et al. Gonococcal lipooligosaccharide suppresses HIV infection in human primary macrophages through induction of innate immunity. J. Infect. Dis. 194, 751-759 (2006).

166. Remmele, C. W. et al. Transcriptional landscape and essential genes of Neisseria gonorrhoeae. Nucleic Acids Res. 42, 10579-10595 (2014)

167. Lee, E. H. \& Shafer, W. M. The farAB-encoded efflux pump mediates resistance of gonococci to long chained antibacterial fatty acids. Mol. Microbiol. 33 , 839-845 (1999)

168. Lee, E. H., Rouquette-Loughlin, C., Folster, J. P. \& Shafer, W. M. FarR regulates the farAB-encoded efflux pump of Neisseria gonorrhoeae via an MtrR regulatory mechanism. J. Bacteriol. 185, 7145-7152 (2003).

169. Warner, D. M., Folster, J. P., Shafer, W. M. \& Jerse, A. E. Regulation of the MtrC-MtrD-MtrE effluxpump system modulates the in vivo fitness of Neisseria gonorrhoeae. J. Infect. Dis. 196 1804-1812 (2007)

170. Seib, K. L. et al. Characterization of the OxyR regulon of Neisseria gonorrhoeae. Mol. Microbiol. 63, 54-68 (2007).

171. Overton, T. W. et al. Coordinated regulation of the Neisseria gonorrhoeae-truncated denitrification pathway by the nitric oxide-sensitive repressor, NsrR, and nitrite-insensitive NarQ-NarP. J. Biol. Chem. 281 , 33115-33126 (2006).

172. Wu, H. J. et al. PerR controls Mn-dependent resistance to oxidative stress in Neisseria gonorrhoeae. Mol. Microbiol. 60, 401-416 (2006).

173. Gunesekere, I. C. et al. Comparison of the RpoHdependent regulon and general stress response in Neisseria gonorrhoeae. J. Bacteriol. 188 , 4769-4776 (2006). 


\section{REVIEWS}

174. Gangaiah, D. et al. Both MisR (CpxR) and MisS (CpxA) are required for Neisseria gonorrhoeae infection in a murine model of lower genital tract infection. Infect. Immun. 85, e00307-17 (2017)

175. Yu, C., McClure, R., Nudel, K., Daou, N. \& Genco, C. A Characterization of the Neisseria gonorrhoeae iron and Fur regulatory network. J. Bacteriol. 198, 2180-2191 (2016).

176. Tseng, H. J., McEwan, A. G., Apicella, M. A. \& Jennings, M. P. OxyR acts as a repressor of catalase expression in Neisseria gonorrhoeae. Infect. Immun. 71, 550-556 (2003)

177. Kim, J. J., Zhou, D., Mandrell, R. E. \& Griffiss, J. M. Effect of exogenous sialylation of the lipooligosaccharide of Neisseria gonorrhoeae on opsonophagocytosis. Infect. Immun. 60, 4439-4442 (1992).

178. Blom, A. M. \& Ram, S. Contribution of interactions between complement inhibitor C4b-binding protein and pathogens to their ability to establish infection with particular emphasis on Neisseria gonorrhoeae. Vaccine 26 (Suppl. 8), 149-155 (2008).

179. Jarvis, G. A. Analysis of C3 deposition and degradation on Neisseria meningitidis and Neisseria gonorrhoeae. Infect. Immun. 62, 1755-1760 (1994).

180. Yu, Q. et al. Association of Neisseria gonorrhoeae Opa(CEA) with dendritic cells suppresses their ability to elicit an HIV-1-specific T cell memory response. PLOS ONE 8, e56705 (2013).

181. Ison, C. A., Deal, C. \& Unemo, M. Current and future treatment options for gonorrhoea. Sex. Transm. Infect. 89 (Suppl. 4), iv52-iv56 (2013).

Acknowledgements

H.S.S. was supported by the US National Institutes of Health (NIH) grant R37-AI033493. S.J.Q. was partially supported by NIH grant T32-AI0007476.

\section{Author contributions}

S.J.Q. and H.S.S. contributed to researching data for the article. S.J.Q. and H.S.S. substantially contributed to the discussion of content. S.J.Q. and H.S.S. wrote the article. S.J.O. and H.S.S. reviewed and edited the manuscript before submission.

\section{Competing interests statement}

The authors declare no competing interests.

\section{Publisher's note}

Springer Nature remains neutral with regard to jurisdictional claims in published maps and institutional affiliations.

\section{Reviewer information}

Nature Reviews Microbiology thanks M. A. Apicella, S. GrayOwen and M. W. Russell for their contribution to the peer review of this work. 